\title{
Bridgehead nitrogen heterocycles which contain the quinazoline moiety - synthesis and cycloaddition of 1,2-dihydroquinazoline 3-oxides
}

Frances Heaney, ${ }^{* a}$ Tomas McCarthy, ${ }^{a}$ Mary Mahon ${ }^{b}$ and V. McKee

a Department of Chemistry, National University of Ireland, Maynooth, Ireland

${ }^{b}$ Department of Chemistry, University of Bath, Bath, UK BA2 7 AY

${ }^{c}$ Chemistry Department, Loughborough University, Loughborough, Leicestershire, UK LE11 $3 T U$

Received 30th August 2005, Accepted 14th October 2005

First published as an Advance Article on the web 16th November 2005

A novel synthesis of 1,2-disubstituted 1,2-dihydroquinazoline 3-oxides 8 and the first ever examples of 1,3-dipolar trapping of these nitrones to homonuclear dipolarophiles is described. The new dipoles $\mathbf{8}$ reacted with $N$-methyl maleimide, generating diastereomeric adducts 14-16. In the reaction between 8 and dimethyl acetylenedicarboxylate, primary cycloadducts $\mathbf{1 7}$ and/or stable rearrangement products, azomethine ylides 18, are formed depending on the substitution pattern of the dipole. The structure of $18 \mathrm{c}$ is unambiguously assigned by X-ray crystallographic analysis. An X-ray crystal structure determination is also presented for the cyclopropylisoxazoloquinazoline $\mathbf{2 2}$ formed by a $[3+2]$ addition of $\mathbf{8 a}$ to $\mathbf{2 1}$, the dimethyl acetylenedicarboxylate tetramer.

\section{Introduction}

In connection with our studies on the formation of nitrones from oximes, ${ }^{1-3}$ we now report the preparation of 1,2 -disubstituted 1,2-dihydroquinazoline 3-oxides from $o$-aminoarylaldoximes.

Prior to the current work there have been no reported examples of the formation of aldonitrones of the 1,2-dihydroquinazoline skeleton; however, several examples of related ketonitrones and aminonitrones are known. Cyclocondensations between 2aminobenzo(aceto)phenone oximes and dichloroethyl ethers, ${ }^{4}$ chloroethylacetoacetate, ${ }^{5}$ ketones ${ }^{6}$ or aldehydes ${ }^{7,8}$ have furnished variously substituted 1,2-dihydroquinazoline ketonitrones. The 1,2,5,6-tetrahydropyrimidine 3-oxides $\mathbf{1}$, monocyclic analogues of quinazoline 3-oxides, have been prepared by cyclocondensation of $\beta$-benzylaminoketoximes with aldehydes. ${ }^{9}$

Corresponding aminonitrones have been formed from $o$ aminobenzamidoximes by cyclocondensation either to imines formed in situ upon reaction with an aldehyde ${ }^{10}$ or to transient iminium ions generated by dehydrogenation [Hg(II), EDTA] of a tertiary amine. ${ }^{11,12}$ The same laboratory have also reported formation of the tricyclic $N$-oxides 3 with a perhydro-pyrido or -pyrrolo ring fused to the 1,2- $a$ edge of the quinazoline nucleus. The cyclic nitrones were generated, in the presence of $\mathrm{Hg}(\mathrm{II})$ EDTA complex, from appropriately substituted benzaldoximes in a type of intramolecular Mannich reaction, ${ }^{13-15}$ for some substrates, e.g. 2, lactams $\mathbf{4}$ accompany the cyclic nitrones $\mathbf{3}$ as minor reaction products.

\section{Results and discussion}

\section{Substrate preparation}

The targeted $o$-aminobenzaldehydes $\mathbf{6 a}, \mathbf{b}$ and $\mathbf{c}$ were prepared by a nickel catalysed addition of the appropriate organozinc reagent to 2,1-benzisoxazole 5 as described in the literature. ${ }^{16}$ The $p-\mathrm{CNC}_{6} \mathrm{H}_{4}$ analogue $\mathbf{6 d}$ was prepared in $77 \%$ yield in a parallel manner. During the synthesis of $\mathbf{6 c}$ and $\mathbf{6 d}$, by-products isolated in 12 and $6 \%$ yield respectively are identified as the $4,4^{\prime}$-disubstituted biphenyl derivatives $9 \mathbf{a}$ and $\mathbf{9 b}{ }^{17}$

Reaction between the $o$-aminobenzaldehydes $\mathbf{6}$ and $\mathrm{NH}_{2} \mathrm{OH} \cdot \mathrm{HCl}\left(\mathrm{C}_{5} \mathrm{H}_{5} \mathrm{~N}, \mathrm{EtOH}, 80^{\circ} \mathrm{C}, 12 \mathrm{~h}\right)$ proceeded smoothly to afford the oximes 7 as single geometrical isomers in solution in $\mathrm{CDCl}_{3}$. Oximes $\mathbf{7 a}, \mathbf{b}$ and $\mathbf{d}$ were isolated in good yields as crystalline solids. The yield of $\mathbf{7 c}$ was somewhat lower with the desired oxime $(60 \%)$ being accompanied by 2 -methoxyacridine $(11 \%)$. The formation of both 2- and 4-methoxyacridine following TFA treatment of 2methoxydiphenylamine 2-carboxaldehyde 6c has previously
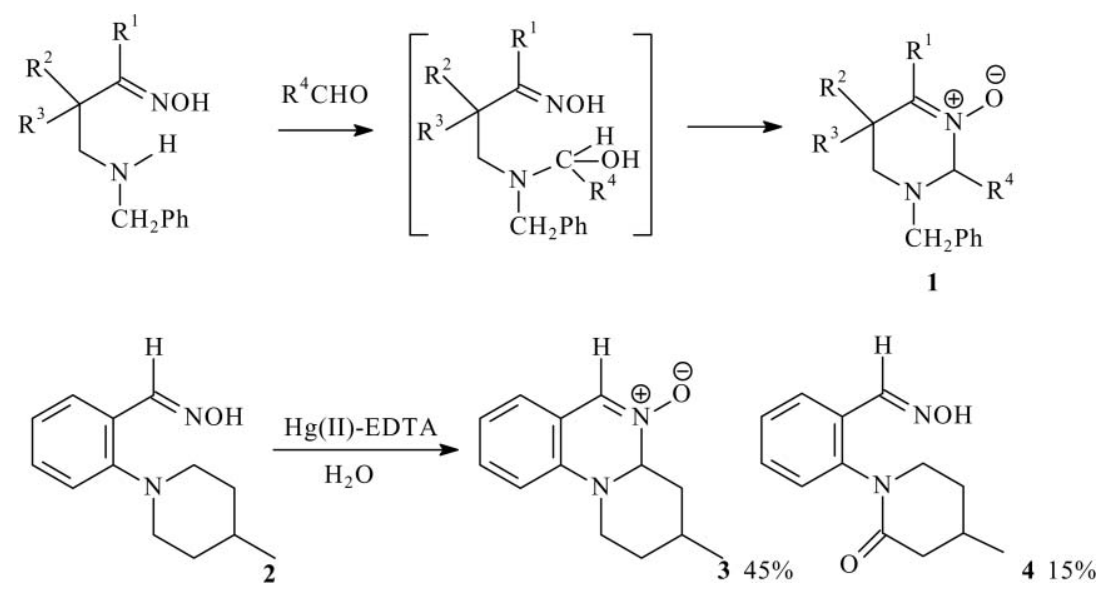


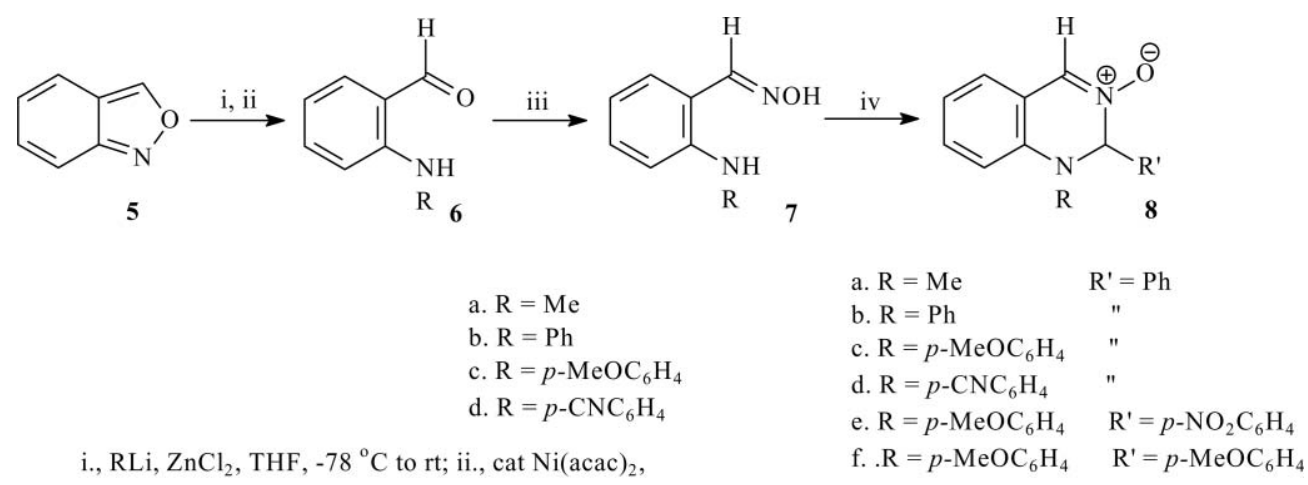

iii., $\mathrm{NH}_{2} \mathrm{OH} . \mathrm{HCl}, \mathrm{C}_{5} \mathrm{H}_{5} \mathrm{~N}$, EtOH, $80^{\circ} \mathrm{C}, 12$ h; iv., $\mathrm{R}^{\prime} \mathrm{CHO}$

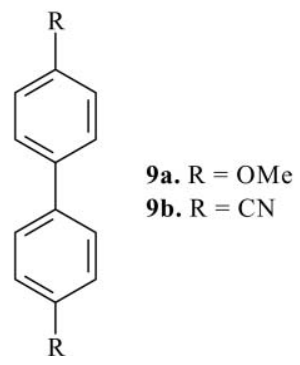

been reported. ${ }^{16}$ It is likely that the pyridinium hydrochloride generated during the oximation of $\mathbf{6 c}$ is responsible for inducing cyclisation of unreacted aldehyde, so leading to the acridine byproduct.

Cyclocondensation of the $o$-aminobenzaldoximes $7 \mathbf{a}$ and $\mathbf{b}$ with benzaldehyde was initially attempted under conditions previously found to be successful for analogous amino oximes, viz. fridge temperature in solution in $\mathrm{Et}_{2} \mathrm{O} / \mathrm{EtOH}^{10}{ }^{10}$ However, no reaction occurred between $7 \mathbf{a}$ and $\mathbf{b}$ and benzaldehyde at $4{ }^{\circ} \mathrm{C}$. Upon heating the reactants in boiling $\mathrm{EtOH}$, evidence for the formation of $\mathbf{8 a}$ and $\mathbf{b}$ could be seen in the ${ }^{1} \mathrm{H}$ NMR spectrum of the crude reaction mixture; however, the desired reaction was overshadowed by the formation of a number of ethoxy addition products. Successful generation of the quinazoline 3-oxides 8a-d resulted when the components were heated at $80^{\circ} \mathrm{C}$ using benzaldehyde ( 5 equiv.) as reactant and solvent. Reaction duration and product yield varied with the nature of the amino substituent. The $N$-methyl- and $N$ - $(p$ methoxyphenyl)-substituted substrates $7 \mathbf{a}$ and $7 \mathbf{c}$ reacted fastest reaching completion after $16 \mathrm{~h}$; the $N$-phenyl substrate $7 \mathbf{b}$ required $72 \mathrm{~h}$ for full reaction whilst the $p$-cyanophenyl group on 7d significantly retarded progress and reaction had only reached $77 \%$ oxime conversion after 72 h. The quinazoline 3-oxides 8 were isolated by flash column chromatography.

In an effort to probe the probe the sensitivity of the reaction to the nature of the aldehyde component, condensation between 2-(4-methoxyanilino)benzaldehyde oxime $7 \mathbf{c}$ and either $p$ nitrobenzaldehyde or $p$-methoxybenzaldehyde was investigated. Both nitrobenzaldehyde and the oxime remained solids at $80^{\circ} \mathrm{C}$ and ${ }^{1} \mathrm{H}$ NMR spectral analysis confirmed that no reaction occurred between the components at this temperature. At $120^{\circ} \mathrm{C}$ the reactants formed a molten solid, and following $4 \mathrm{~h}$ of heating complete conversion to 8e was observed. The same oxime 7c reacts with $p$-methoxybenzaldehyde rather sluggishly at $80{ }^{\circ} \mathrm{C}$ and after $236 \mathrm{~h} \mathbf{8 f}$ isolated in $49 \%$ yield, starting oxime was recovered in $32 \%$ yield. Reaction progressed faster at $120{ }^{\circ} \mathrm{C}$ having reached the same level of oxime conversion after $84 \mathrm{~h}$, when ${ }^{1} \mathrm{H}$ NMR spectral data indicated a $1: 2$ mixture of oxime : nitrone $7 \mathrm{c}: \mathbf{8 f}$.

In order to permit direct comparison between the nature of the $o$-amino substituent on the oxime substrate 7 and the condensing aldehyde, the reaction of $7 \mathbf{c}$ with benzaldehyde was repeated at $120^{\circ} \mathrm{C}$. After $6.5 \mathrm{~h}$ of heating almost quantative conversion to $8 \mathrm{c}$ was found. Thus, as expected, the more electron-rich the $o$-amino oxime substituent and the more electron-poor the condensing aldehyde the faster the cyclocondensation reactions leading to 8 .

\section{Cycloaddition to quinazoline 3-oxides}

The reactivity of 1,2-dihydroquinazoline 3-oxides has not been extensively investigated. Aromatisation, ${ }^{18,19}$ ring expansion ${ }^{5,20}$ and hydrolysis ${ }^{6}$ of ketonitrones has been demonstrated, whilst Dimroth rearrangements to isomeric 1,2-dihydro-4-quinaxolone oximes are facile with aminonitrones. ${ }^{10}$ The trapping of 10a with phenyl isocyanate represents the only reported example of 1,3-dipolar cycloaddition to 1,2-dihydroquinazoline-3-oxides. ${ }^{13}$ The same heterodipolarophile trapped the amidonitrone $\mathbf{1 0 b}^{11}$ furnishing 11b. Aside from these two reactions there has been no exemplification of the 1,3-dipolar character of the 1,2-dihydroquinazoline 3-oxide skeleton. However, a related reaction is the trapping of the tetrahydropyrimidine $N$-oxide 12 with dimethyl acetylenecarboxylate $\left(4 \mathrm{~h}, \mathrm{CHCl}_{3}\right)$, furnishing $13(75 \%)$ as a single diastereomeric cycloadduct. ${ }^{9}$<smiles>[R]C1=[N+]([O-])C2CCCCN2c2ccccc21</smiles>

10a. $\mathrm{R}=\mathrm{H}$

10b. $\mathrm{R}=\mathrm{NMe}_{2}$
$\mathrm{PhNCO}$<smiles>[R]C12c3ccccc3N3CCCCC3N1OC(=O)N2c1ccccc1</smiles>

11<smiles>[O-][N+]1=C(Cc2ccccc2)C(c2ccccc2)CN(Cc2ccccc2)C1</smiles><smiles>COCC#CC(=O)OC</smiles>

$\mathrm{CH}_{2} \mathrm{Ph}$

12<smiles>CC(=O)C1=C(C(C)=O)C2(C)C(c3ccccc3)CN(Cc3ccccc3)CN2O1</smiles>

13 
<smiles>[R]C1Nc2ccccc2C=[N+]1[O-]</smiles>

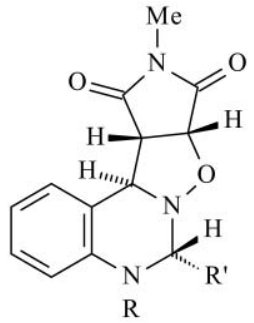

14<smiles>[R]C1Nc2ccccc2[C@@H]2[C@@H]3C(=O)N(C)C(=O)[C@H]3ON1[C@H]2[R]</smiles>

15<smiles>[R]C1([R])Nc2ccccc2[C@@H]2[C@@H]3C(=O)N(C)C(=O)[C@H]3ON21</smiles>

16

a. $\mathrm{R}=\mathrm{Me}, \mathrm{R}^{\prime}=\mathrm{Ph} ; \mathrm{b} \cdot \mathrm{R}=\mathrm{Ph}, \mathrm{R}^{\prime}=\mathrm{Ph} ; \mathrm{c} . \mathrm{R}=p-\mathrm{MeOC}_{6} \mathrm{H}_{4}, \mathrm{R}^{\prime}=\mathrm{Ph}$

d. $\mathrm{R}=p-\mathrm{CNC}_{6} \mathrm{H}_{4}, \mathrm{R}^{\prime}=\mathrm{Ph}$; e. $\mathrm{R}=p-\mathrm{MeOC}_{6} \mathrm{H}_{4}, \mathrm{R}^{\prime}=p-\mathrm{NO}_{2} \mathrm{C}_{6} \mathrm{H}_{4}$

Reaction between the quinazoline 3-oxides $\mathbf{8}$ and $\mathrm{N}$-methyl maleimide proceeded under mild conditions $\left(\mathrm{CH}_{2} \mathrm{Cl}_{2}, \mathrm{rt}, 18 \mathrm{~h}\right)$ to afford, with varying selectivity, diastereomeric cycloadducts 14-16. The relative stereochemistry of the adducts arising from reaction of $\mathbf{8 b}$ was established by nuclear Overhauser effect difference spectra (nOeds) studies and that of the others by analogy.

The major diastereomer 14b (55\%) displays a 3.6\% enhancement on the signal for $\mathrm{H}-11 \mathrm{~b}$ following irradiation of $\mathrm{H}-11 \mathrm{a}$, no cross-ring enhancements between H-6 and any of H-8a, H-11a or $\mathrm{H}-11 \mathrm{~b}$ are found. The intermediate isomer $\mathbf{1 5 b}(35 \%)$ displays a $2.8 \%$ enhancement on the signal representing $\mathrm{H}-11 \mathrm{~b}$ following irradiation of H-11a, and mutual cross-ring enhancements are observed between $\mathrm{H}-6$ and $\mathrm{H}-11 \mathrm{~b}$. The minor isomer $\mathbf{1 6 b}(10 \%)$ shows no cross-ring enhancements between $\mathrm{H}-6$ and any of $\mathrm{H}-$ $8 \mathrm{a}, \mathrm{H}-11 \mathrm{a}$ or $\mathrm{H}-11 \mathrm{~b}$; however, there is an $8.5 \%$ enhancement on the signal for $\mathrm{H}-11 \mathrm{a}$ following irradiation of $\mathrm{H}-11 \mathrm{~b}$. These observations imply that the major product $\mathbf{1 4 b}$ is the product of an exo-addition of the dipolarophile to the top face of the dipole. $\uparrow$ The intermediate adduct $\mathbf{1 5 b}$ arises from an exoaddition of the dipolarophile to the lower face of the dipole. The minor adduct $\mathbf{1 6} \mathbf{b}$ is a product of an endo-addition to the top face of the dipole.

Within each family of diastereomeric cycloadducts 14-16a-e a distinct pattern can be identified in the ${ }^{1} \mathrm{H}$ NMR resonance positions for the non-aromatic protons viz. H-6, H-8a, H-11a and $\mathrm{H}-11 \mathrm{~b}$. Thus, for all the major adducts $14, \mathrm{H}-11 \mathrm{a}$ resonates between 3.77 and 3.84 ppm; $\mathrm{H}-11 \mathrm{~b}$ resonates between 4.47 and $4.58 \mathrm{ppm}$; and $\mathrm{H}-8 \mathrm{a}$ resonates between 4.77 and $4.87 \mathrm{ppm}$. For the adducts $\mathbf{1 5}$ the $\mathrm{H}-11 \mathrm{~b}$ signal experiences a downfield shift of $c a .0 .7 \mathrm{ppm}$ with respect to the corresponding signal in the diastereomers 14. The proton with the most significant difference in resonance position between adducts $\mathbf{1 4}$ and $\mathbf{1 6}$ is $\mathrm{H}-11 \mathrm{a}$ which appears $c a$. $0.4 \mathrm{ppm}$ downfield in the adducts $\mathbf{1 6}$ with respect to $\mathbf{1 4}$.

The similarity between the ${ }^{1} \mathrm{H}$ NMR spectral data of the adducts 14a,c-e, 15a,c-e and 16a,c-e with 14b, 15b and $16 b$ suggests that each diastereomer has a favoured conformation in solution and permits extrapolation of the nOeds data acquired for the latter to complete the assignment of the relative stereochemistry of the former.

Reaction between the dipoles $\mathbf{8}$ and dimethyl acetylenedicarboxylate also proceeded to completion upon stirring an equimolar ratio of the reactants in $\mathrm{CH}_{2} \mathrm{Cl}_{2}$ at $\mathrm{rt}$ for $18 \mathrm{~h}$. The reaction products varied with the nature of the $N-1$ substituent on the dipole. All the $N-1$ aryl substrates $\mathbf{8 b}-\mathbf{e}$ reacted to afford primary cycloaddition products $\mathbf{1 7} \mathbf{b}-\mathbf{e}$, accompanied, in the cases of $8 \mathbf{b}$ and $8 \mathbf{c}$, by secondary products $18 \mathbf{b}, \mathbf{c}$. The $N-1$ methyl derivative $\mathbf{8 a}$ reacted rather differently.

$\dagger$ The 3-Si,4-Re face of the quinazoline 3-oxides is designated as the top face.
Analysis of the ${ }^{1} \mathrm{H}$ NMR spectral data of the crude mixture following reaction between $\mathbf{8 c}$ and dimethyl acetylenedicarboxylate indicated the presence of two new reaction products. Purification by flash column chromatography afforded pure samples of each. The major product $(32 \%)$, a red powder, was characterised as the primary cycloaddition product $17 \mathbf{c}$. No enhancements were observed on either $\mathrm{H}-5$ or $\mathrm{H}-10 \mathrm{~b}$ upon irradiation of the other, thus it is tentatively proposed that the relative stereochemistry of $17 \mathbf{c}$ is as shown in the diagram.

The minor product $(24 \%)$, a red crystalline solid, was unambiguously identified by single crystal X-ray analysis as the stable bicyclic azomethine ylide 18c (Fig. 1). There is

(a)
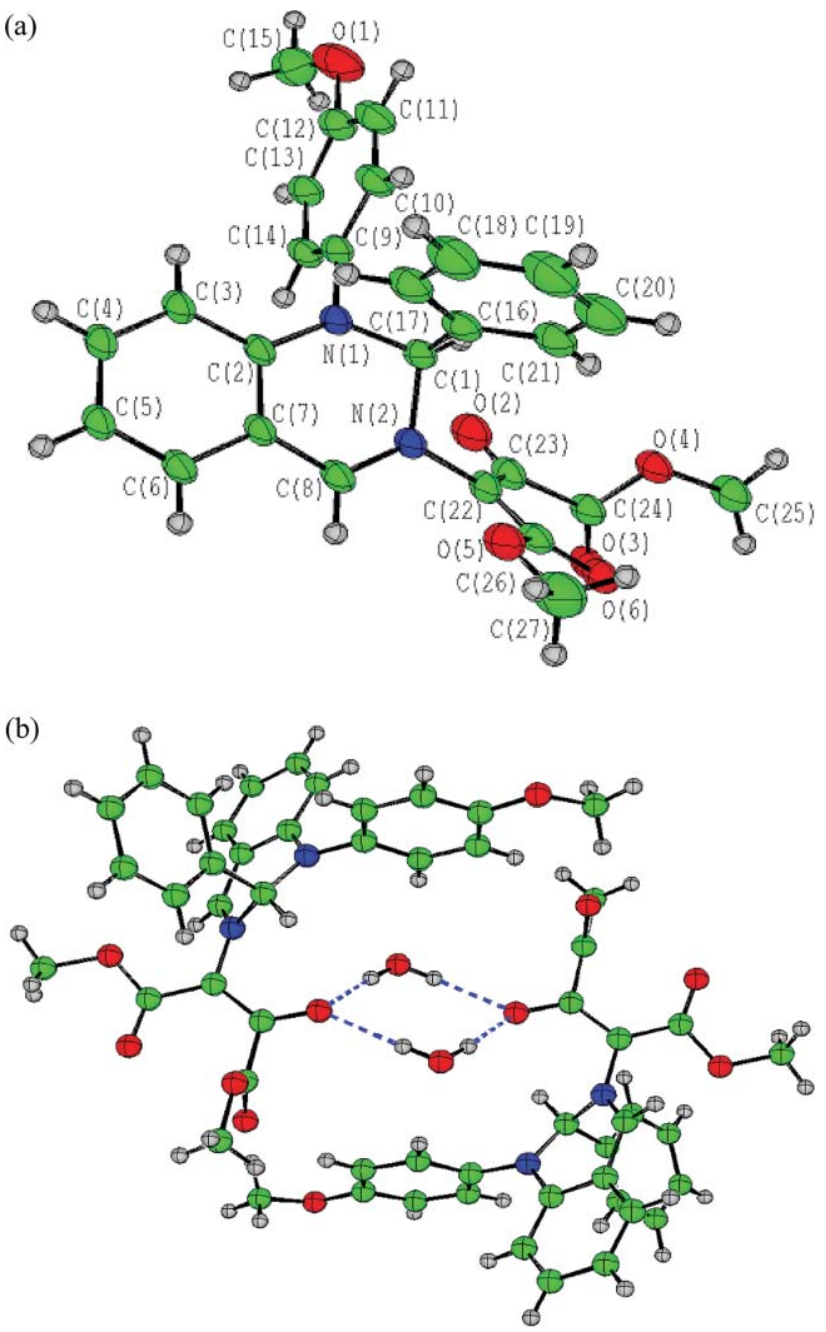

Fig. 1 X-Ray crystallographic presentation of 18c: (a) showing monomer unit, and (b) showing water of crystallization and H-bonded dimers. 
water of crystallization in the ylide structure. The crystal structure contains $\mathrm{H}$-bonded dimers with the water molecules $\mathrm{H}$-bonded to the keto-carbonyl oxygen atom $\mathrm{O}(2)$ [Fig. 1(b)]. The determination of the structure of $\mathbf{1 8 c}$ is significant since, in as far as we can gather to-date, only a handful of azomethine ylides have been characterised in this way. ${ }^{21-27}$ The $\mathrm{C}-\mathrm{N}$ bonds of the dipolar system differ significantly in length with the $\mathrm{N}(2)$ $\mathrm{C}(22)$ bond [1.443(2) $\AA$ ] being close to a typical ${ }^{\mathrm{sp}^{3}} \mathrm{~N}-\mathrm{C}^{\mathrm{sp}}{ }^{3}$ bond $(1.48 \AA)$ whilst the $\mathrm{N}(2)-\mathrm{C}(8)$ bond $[1.298(3) \AA]$ is slightly shorter than a typical $\mathrm{C}=\mathrm{N}$ double bond. These measurements suggest strong bond localisation, a feature characteristic of those isolable azomethine ylides for which X-ray structural analysis has been performed. ${ }^{21-27} \mathrm{~A}$ twist angle of $64.5^{\circ}$ between the planes defined by $\mathrm{C}(8)-\mathrm{N}(2)-\mathrm{C}(1)$ and $\mathrm{C}(22)-\mathrm{C}(23)-\mathrm{C}(26)$ further testifies to the low degree of conjugative interaction between the iminium and the anionic functionalities of the dipolar system. The lack of $\pi$-conjugation coupled with the non-planarity of the dipole unit suggest a role for steric features and/or electronic interactions at the ionic centres as the driving force(s) for stabilisation of 18c. These structural parameters also call into question the classification of $\mathbf{1 8 c}$ as a 1,3-dipole and experiments are currently on-going to probe this hypothesis.

Azomethine ylides are widely recognised as key intermediates in a variety of thermal rearrangements of 4-isoxazolines, ${ }^{28}$ however, aside from when the $\mathrm{C}=\mathrm{N}$ bond is part of an aromatic system, the ylides have seldom been observed to be isolable. Exceptions include an example where the $\mathrm{C}=\mathrm{N}$ moiety is part of a 3,4-dihydroisoquinoline structure ${ }^{29}$ or where the $\mathrm{C}$ atom of the $\mathrm{C}=\mathrm{N}$ unit bears a phenyl or styryl group. ${ }^{30,31}$ Eberbach and coworkers have recently reported the first examples of isoxazolines which transform to stable, isolable, azomethine ylides having stabilising groups only at one end of the dipole. ${ }^{21,32}$ To the best of our knowledge, compounds $\mathbf{1 8}$ represent a new type of stable azomethine ylide where the $\mathrm{C}=\mathrm{N}$ moiety is part of a dihydroquinazoline ring system.

The $N-1$ phenyl dipole $\mathbf{8 b}$ reacted in a parallel fashion to that observed for $\mathbf{8 c}$ and the primary cycloaddition product $\mathbf{1 7} \mathbf{b}$ was isolated with the stable dipole 18b in 63 and $14 \%$ yield respectively. It was subsequently found that smooth conversion between $\mathbf{1 7 b}, \mathbf{c}$ and $\mathbf{1 8 b}, \mathbf{c}$ could be achieved upon stirring the primary adducts in solution in $\mathrm{MeOH}$.

Reaction between dipoles $8 \mathbf{d}$ and $\mathbf{8 e}$ and dimethyl acetylenedicarboxylate furnished only the primary cycloaddition products 17d (quantative yield) and $\mathbf{1 7 e}(79 \%)$. The $N-1$ methyl derivative 8a reacted rather differently from its aryl counterparts with dimethyl acetylenedicarboxylate. No primary cycloaddition products were found in the reaction mixture. ${ }^{1} \mathrm{H}$ NMR spectral analysis indicated the presence of one main product together with a number of minor products. Purification by flash column chromatography gave a pure sample of the main product, a red crystalline solid, characterised as the stable azomethine ylide 18a $(60 \%)$. A number of minor products with varying stability were also isolated but we are not in a position to confirm their structure.

Significantly, during the isolation of $\mathbf{1 8 a}$ a colourless crystalline solid fell from the early column fractions following standing at $\mathrm{rt}$, and this material was subsequently found by X-ray crystal structure analysis to be the cyclopropaisoxazoloquinazoline $22(5 \%)$ shown in Fig. 2. Dimethyl acetylenedicarboxylate is known to undergo spontaneous tetramerisation ${ }^{33}$ and it may be the case that a small amount of the tetramer was indeed present in the starting ester - a situation previously alluded to in the literature. ${ }^{34}$ Whilst [4 + 2]-cycloaddition of cyclopentadienes to the dimethyl acetylenedicarboxylate tetramer have previously been noted ${ }^{35}$ to the best of our knowledge the formation of $\mathbf{2 2}$ represents the first example of a $[3+2]$-cycloaddition to the DMAD tetramer 21. Indeed, the formation of 22 represents only the second publication of a cycloaddition reaction between a nitrone and a cyclopropene moiety ${ }^{36}$ and only the third report on the formation of cyclopropyl fused isoxazolidines. ${ }^{37}$

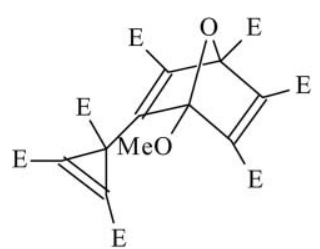

21

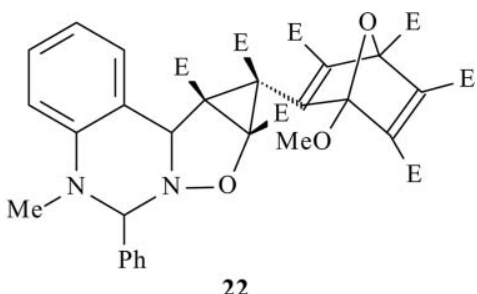

22

$$
\mathrm{E}=\mathrm{CO}_{2} \mathrm{Me}
$$

Rearrangement of 4-isoxazolines to azomethine ylides is well known and it is accepted that mechanistically the transformation proceeds in a concerted fashion by way of an acyl aziridine intermediate. If $\mathbf{1 7}$ rearranges to $\mathbf{1 8}$ directly by way of the aziridine 19 (Fig. 3, Path A) it is difficult to explain the relative stability of the $N-6$ aryl derivatives $17 \mathbf{b}-\mathbf{e}$ with respect to the $N-6$

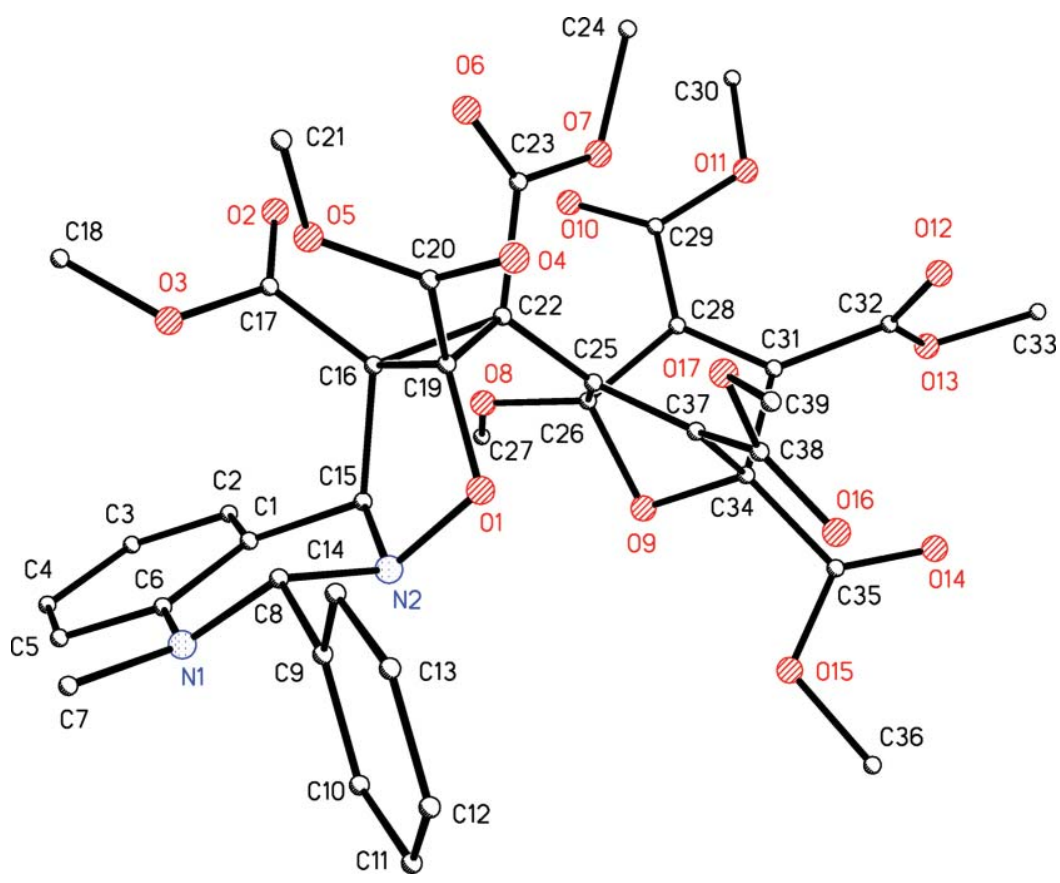

Fig. 2 X-Ray crystallographic presentation of 22. 
<smiles>[R]C1N([O-])c2ccccc2C=[N+]1[O-]</smiles>

8

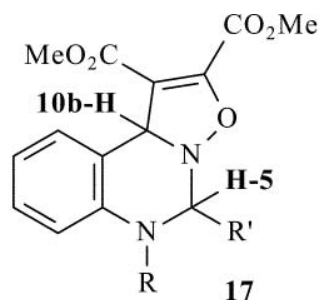

17

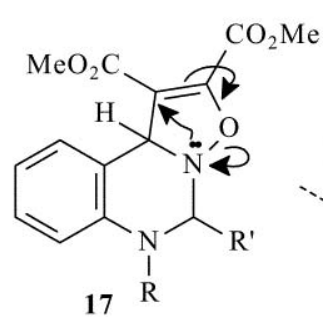

Path A<smiles></smiles>

18

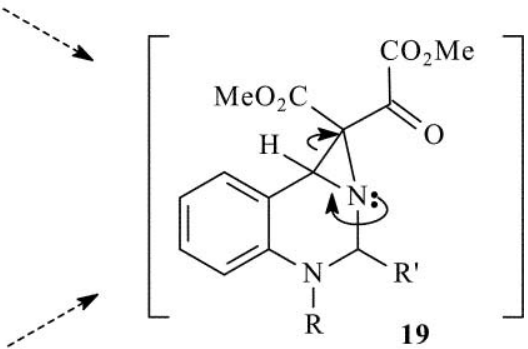

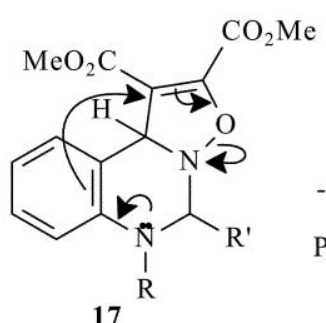

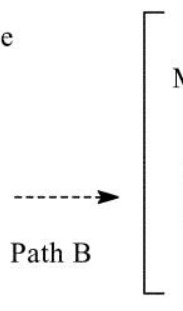

$\mathrm{Me}$<smiles>COc1ccccc1</smiles>

Fig. 3 Proposed mechanistic origin of ylides $\mathbf{1 8}$ from the cycloadducts $\mathbf{1 7}$.

methyl analogue 17a. We suggest that an alternative rearrangement pathway may also operate. A two-step process, shown in Fig. 3 (Path B), invoking 20 as a precursor to the aziridine $\mathbf{1 9}$ accounts for the increase in lability of the isoxazoline ring with increasing electron releasing power on the $N-6$ substituent. The proposal of the intermediary cyclopropyl fused ring skeleton 20 is in parallel with Freeman's ring contraction mechanism for isoxazolobenzodiazepines ${ }^{38}$

\section{Conclusions}

It has been shown that 1,2-dihydroquinazoline 3-oxides 8 are readily prepared from $o$-aminoarylaldoximes and aryl aldehydes in a cyclocondensation reaction. For the first time this family of heterocyclic $N$-oxides have been demonstrated to have 1,3-dipolar character being trapped, under mild reaction conditions, by $N$-methyl maleimide affording the diastereomeric primary cycloaddition products 14-16. In reaction with dimethyl acetylenedicarboxylate the products comprise primary 17 and or secondary 18 products of cycloaddition depending on the nature of the $N-1$ substituent. The dipole $\mathbf{8 a}$ has also been trapped by the cyclopropene moiety of the dimethyl acetylenedicarboxylate tetramer 21.

\section{Experimental}

Melting points were determined on a Stuart Scientific (Bibby) melting point apparatus and are uncorrected. Elemental analyses were performed on a CE-440 analytical instrument. ${ }^{1} \mathrm{H}$ and ${ }^{13} \mathrm{C}$ NMR spectra were recorded using a Bruker NMR spectrometer operating at $300 \mathrm{MHz}$ for ${ }^{1} \mathrm{H}$ and $75 \mathrm{MHz}$ for ${ }^{13} \mathrm{C}$ nuclei. Data were recorded at probe temperatures with, unless otherwise stated, tetramethylsilane as internal reference and deuteriochloroform as solvent; $J$ values are given in Hertz. Flash column chromatography was carried out on silica gel 60
$(0.040-0.063 \mathrm{~nm})$ purchased from Merck, analytical TLC plates were purchased from Merck, aluminium-backed and coated with silica gel $60 \mathrm{~F}_{254}$ indicator. Samples were located by UV illumination using a portable UVtec lamp $(\lambda=254 \mathrm{~nm})$ or by the use of iodine staining. Mass spectra were recorded on a Profile Kratos Analytical instrument. Infrared spectra were recorded on a Perkin Elmer 2000 FT-IR instrument, and samples were prepared as $\mathrm{KBr}$ discs.

\section{Preparation of the 2-aminobenzaldehydes 6}

The substrates 6a $(1.30 \mathrm{~g}, 90 \%), \mathbf{6 b}(1.70 \mathrm{~g}, 86 \%)$ and $\mathbf{6 c}$ $(1.40 \mathrm{~g}, 65 \%)$ were prepared by addition of the appropriate organozinc reagent to 2,1-benzisoxazole by the method of Baum et al. In each case the ${ }^{1} \mathrm{H}$ NMR spectral data agree with those published. ${ }^{16}$ 4'-Methoxy-[1,1'-biphenyl]-4-yl methyl ether 9a $(0.26 \mathrm{~g}, 12 \%)$ was formed as by-product during the preparation of $\mathbf{6 c}$; the ${ }^{1} \mathrm{H}$ NMR spectral data of isolated 9a agree with the literature. ${ }^{17}$

\section{4-(2-Formylanilino)benzonitrile 6d}

To a solution of 4-bromobenzonitrile (3.66 g, $20 \mathrm{mmol})$ in anhydrous THF $\left(25 \mathrm{~cm}^{3}\right)$ under nitrogen at $-78{ }^{\circ} \mathrm{C}$ was added dropwise a solution of $1.6 \mathrm{M} n$-butyllithium in hexane $\left(12.5 \mathrm{~cm}^{3}\right.$, $20 \mathrm{mmol}$ ). The resulting suspension was added rapidly, via a cannula (18 gauge double tipped needle), to a stirred slurry of anhydrous $\mathrm{ZnCl}_{2}(3.41 \mathrm{~g}, 25 \mathrm{mmol})$ in THF $\left(75 \mathrm{~cm}^{3}\right)$ under nitrogen at $0{ }^{\circ} \mathrm{C}$. When the solution had warmed to $\mathrm{rt}, 2,1$ benzisoxazole $(10 \mathrm{mmol})$ in THF $\left(10 \mathrm{~cm}^{3}\right)$ was added followed by anhydrous $\mathrm{Ni}(\mathrm{acac})_{2}(1.00 \mathrm{~g}, 4 \mathrm{mmol})$ in THF $\left(20 \mathrm{~cm}^{3}\right)$. Reaction progress was monitored by periodically working up $1 \mathrm{~cm}^{3}$ aliquots and observing the loss of the starting 2,1benzisoxazole by TLC analysis. Upon completion the reaction was quenched with $6 \mathrm{M} \mathrm{HCl}\left(5 \mathrm{~cm}^{3}\right)$. The reaction mixture was 
poured into water $\left(100 \mathrm{~cm}^{3}\right)$ and extracted with diethyl ether $(2 \times$ $\left.100 \mathrm{~cm}^{3}\right)$. The ether extracts were combined, washed with water $\left(50 \mathrm{~cm}^{3}\right)$ and concentrated under reduced pressure to give the crude product. Purification by flash column chromatography (hexane-diethyl ether; $9: 1$ ) afforded the pure aldehyde as a yellow crystalline solid $(1.70 \mathrm{~g}, 77 \%)$ along with [1,1'-biphenyl]$4,4^{\prime}$-dicarbonitrile $9 \mathbf{b}(0.10 \mathrm{~g}, 6 \%)$. The ${ }^{1} \mathrm{H}$ NMR spectral data of the biphenyl agree with those reported. ${ }^{17}$

6d. $\mathrm{Mp} 102-104^{\circ} \mathrm{C}$ (from EtOH). (Found: $\mathrm{C}, 75.13 ; \mathrm{H}, 4.45$; $\mathrm{N}, 12.46 . \mathrm{C}_{14} \mathrm{H}_{10} \mathrm{~N}_{2} \mathrm{O}$ requires: $\left.\mathrm{C}, 75.68 ; \mathrm{H}, 4.50 ; \mathrm{N}, 12.60 \%\right) . \delta_{\mathrm{H}}$ : $10.21(\mathrm{~s}, 1 \mathrm{H}, \mathrm{NH}), 9.93(\mathrm{~s}, 1 \mathrm{H}, \mathrm{HC}=\mathrm{O}), 7.63(\mathrm{~m}, 3 \mathrm{H}, \mathrm{Ar}-\mathrm{H}$, including 7.62, d, $2 \mathrm{H}, J$ 8.8, $\mathrm{H}-2^{\prime}$ and $\left.\mathrm{H}^{-} 6^{\prime}\right), 7.47(\mathrm{~m}, 2 \mathrm{H}, \mathrm{Ar}-\mathrm{H})$, $7.34\left(\mathrm{~d}, 2 \mathrm{H}, J\right.$ 8.8, ${\mathrm{H}-3^{\prime}}^{\prime}$ and $\left.\mathrm{H}-5^{\prime}\right), 7.02(\mathrm{~m}, 1 \mathrm{H}, \mathrm{Ar}-\mathrm{H}) . \delta_{\mathrm{C}}: 195.0$ $(\mathrm{C}=\mathrm{O}), 145.2,144.9(\mathrm{ArC}-\mathrm{N}), 137.2,136.0,134.0121 .3,120.6$ $(\mathrm{Ar}-\mathrm{CH}), 120.0(\mathrm{C} \equiv \mathrm{N}),(\mathrm{Ar}-\mathrm{CH}), 119.4(\mathrm{ArC}-\mathrm{CHO}), 114.5$ $(\mathrm{Ar}-\mathrm{CH}), 105.9(\mathrm{ArC}-\mathrm{C} \equiv \mathrm{N}) . \mathrm{IR} / \mathrm{cm}^{-1}: v=3424,3108,2844$, 2764, 2219, 1659, 1595, 1529.

\section{General procedure for the formation of 2-aminobenzaldoximes 7}

A solution of the aldehyde $(8 \mathrm{mmol})$, pyridine $(8.1 \mathrm{mmol})$ and $\mathrm{NH}_{2} \mathrm{OH} \cdot \mathrm{HCl}(8.1 \mathrm{mmol})$ were heated in boiling EtOH $\left(50 \mathrm{~cm}^{3}\right)$ for $18 \mathrm{~h}$. Following evaporation of the solvent the residue was taken up in chloroform $\left(50 \mathrm{~cm}^{3}\right)$ and extracted with water $\left(3 \times 40 \mathrm{~cm}^{3}\right)$. The combined organic extracts were dried over $\mathrm{MgSO}_{4}$ and concentrated to yield the crude product which was purified by flash column chromatography or crystallization. 2Methoxyacridine was isolated $(11 \%)$ during preparation of $7 \mathbf{c}$.

2-Methylaminobenzaldehyde oxime $7 \mathbf{a}^{40}$ and 2-anilinobenzaldehyde oxime $\mathbf{7} \mathbf{b}^{41}$ are known compounds; ${ }^{1} \mathrm{H}$ NMR spectral data of the prepared samples agree with published data.

2-(4-Methoxyanilono)benzaldehyde oxime 7c. White crystalline solid $(1.62 \mathrm{~g}, 60 \%), \mathrm{mp} 90-92{ }^{\circ} \mathrm{C}$ (from diethyl ether and hexane). (Found: $\mathrm{C}, 68.90 ; \mathrm{H}, 5.83 ; \mathrm{N}, 11.35 . \mathrm{C}_{14} \mathrm{H}_{14} \mathrm{~N}_{2} \mathrm{O}_{2}$ requires: $\mathrm{C}, 69.42 ; \mathrm{H}, 5.42 ; \mathrm{N}, 11.62 \%) . \delta_{\mathrm{H}}: 8.63(\mathrm{br}, \mathrm{s}, 1 \mathrm{H}$, $\mathrm{NH} / \mathrm{OH}), 8.29(\mathrm{~s}, 1 \mathrm{H}, \mathrm{HC}=\mathrm{N}), 7.16(\mathrm{~m}, 4 \mathrm{H}, \mathrm{Ar}-\mathrm{H}$ including $\mathrm{d}$ at 7.16, 2H, $J 8.8,{\mathrm{H}-3^{\prime}}^{\prime}$ and $\left.\mathrm{H}-5^{\prime}\right), 7.00(\mathrm{~m}, 1 \mathrm{H}, \mathrm{Ar}-\mathrm{H}), 6.90$ $\left(\mathrm{d}, 2 \mathrm{H}, J\right.$ 8.8, $\mathrm{H}-2^{\prime}$ and $\left.\mathrm{H}_{-} 6^{\prime}\right), 6.73(\mathrm{~m}, 1 \mathrm{H}, \mathrm{Ar}-\mathrm{H}), 3.82(\mathrm{~s}, 3 \mathrm{H}$, $\left.\mathrm{OCH}_{3}\right) . \delta_{\mathrm{C}}: 156.8(\mathrm{ArC}-\mathrm{O}), 154.1(\mathrm{HC}=\mathrm{N}), 145.9,134.2(\mathrm{ArC}-$ N) $133.2,130.9,125.9(\mathrm{Ar}-\mathrm{CH}), 117.3\left(\mathrm{ArC}-2^{\prime}\right.$ and $\left.6^{\prime}\right) 115.1$ $(\mathrm{ArC}-\mathrm{C}=\mathrm{NOH}), 115.0,112.9(\mathrm{Ar}-\mathrm{CH}), 56.0\left(\mathrm{OCH}_{3}\right)$. 42,43

2-Methoxyacridine. Colourless crystalline solid (0.13 g, $11 \%$ ) mp $89-91{ }^{\circ} \mathrm{C}$ (from diethyl ether and hexane). $\delta_{\mathrm{H}}: 8.56$ $(\mathrm{s}, 1 \mathrm{H}, \mathrm{C}=\mathrm{CH}), 8.19(\mathrm{~d}, 1 \mathrm{H}, J$ 8.6, Ar-H), $8.12(\mathrm{~d}, 1 \mathrm{H}, J$ 8.6, Ar-H), $7.92(\mathrm{~d}, 1 \mathrm{H}, J$ 8.4, Ar-H), $7.70(\mathrm{~m}, 1 \mathrm{H}, \mathrm{Ar}-\mathrm{H}), 7.48(\mathrm{~m}$, 2H, Ar-H), 7.11 (d, 1H, J 2.6, Ar-H), 3.95 (s, 3H, OMe).

4- $\{$ 2-[(Hydroxyimino)methyl]anilino $\}$ benzonitrile 7d. Yellow crystalline solid (1.00 g, 72\%), mp $166-170{ }^{\circ} \mathrm{C}$ (from toluene). (Found: C, 71.08; H, 4.74; N, 17.18. $\mathrm{C}_{14} \mathrm{H}_{11} \mathrm{~N}_{3} \mathrm{O}$ requires: $\mathrm{C}$, $70.88 ; \mathrm{H}, 4.64 ; \mathrm{N}, 17.72 \%$ ). $\delta_{\mathrm{H}}: 9.06$ (br, s, $\left.1 \mathrm{H}, \mathrm{NH} / \mathrm{OH}\right), 8.26$ $(\mathrm{s}, 1 \mathrm{H}, \mathrm{HC}=\mathrm{N}), 7.53(\mathrm{~m}, 2 \mathrm{H}, \mathrm{Ar}-\mathrm{H}), 7.44(\mathrm{~m}, 2 \mathrm{H}, \mathrm{Ar}-\mathrm{H}), 7.30$ $(\mathrm{m}, 2 \mathrm{H}, \mathrm{Ar}-\mathrm{H}), 7.21(\mathrm{~m}, 2 \mathrm{H}, \mathrm{Ar}-\mathrm{H}), 6.98(\mathrm{~m}, 1 \mathrm{H}, \mathrm{Ar}-\mathrm{H}) . \delta_{\mathrm{C}}$ : $153.5(\mathrm{CH}=\mathrm{N}), 146.5,141.1(\mathrm{ArC}-\mathrm{N}), 134.1,133.3,130.7,121.2$ $(\mathrm{Ar}-\mathrm{CH}), 119.9,118.9(\mathrm{C} \equiv \mathrm{N}$ and $\mathrm{ArC}-\mathrm{C}=\mathrm{N}), 118.2,116.6(\mathrm{Ar}-$ $\mathrm{CH}), 103.9(\mathrm{ArC}-\mathrm{C} \equiv \mathrm{N}) . \mathrm{IR} / \mathrm{cm}^{-1}: v=3335,2222,1625,1610$, $1591,1528$.

\section{1-Methyl-2-phenyl-1,2-dihydroquinazolin-3-ium-3-olate 8a}

2-Methylaminobenzaldehyde oxime 7a (6.8 mmol, $1.00 \mathrm{~g})$ was stirred with benzaldehyde ( $34 \mathrm{mmol}, 3.60 \mathrm{~g}$ ) at $80^{\circ} \mathrm{C}$ for $16 \mathrm{~h}$. Purification by flash column chromatography (diethyl ether) afforded 8a as a green solid $(1.30 \mathrm{~g}, 80 \%), \mathrm{mp} 132-136^{\circ} \mathrm{C}$ (from diethyl ether and hexane). (Found: C, 75.61; H, 5.83; N, 11.90. $\mathrm{C}_{15} \mathrm{H}_{14} \mathrm{~N}_{2} \mathrm{O}$ requires: $\left.\mathrm{C}, 75.63 ; \mathrm{H}, 5.88 ; \mathrm{N}, 11.76 \%\right) . \delta_{\mathrm{H}}: 7.68(\mathrm{~s}$, $1 \mathrm{H}, \mathrm{HC}=\mathrm{N}), 7.35(\mathrm{~m}, 6 \mathrm{H}, \mathrm{Ar}-\mathrm{H}), 7.04(\mathrm{dd}, 1 \mathrm{H}, J 7.6$ and 1.5, Ar-H), 6.81 (ddd, $1 \mathrm{H}, J$ 8.5, 8.5 and 0.9, Ar-H), $6.68(\mathrm{~d}, 1 \mathrm{H}, J$ 8.2, $\mathrm{Ar}-\mathrm{H}), 5.95$ (s, $1 \mathrm{H}, \mathrm{H}-2), 2.93$ (s, 3H, Me). $\delta_{\mathrm{C}}: 141.0(\mathrm{C}-8 \mathrm{a})$,
$135.5\left(\mathrm{C}-1^{\prime}\right), 132.0(\mathrm{CH}=\mathrm{N}), 131.8,130.1,129.2,129.0,128.8$, 127.0, 126.4, 119.6 (Ar-CH), 116.6 (C-4a), 111.7 (Ar-CH), 86.9 (C-2), $36.1(\mathrm{NMe})$.

\section{1,2-Diphenyl-1,2-dihydroquinazolin-3-ium-3-olate 8b}

2-Phenylaminobenzaldehyde oxime $7 \mathbf{b}(1.90 \mathrm{~g}, 9 \mathrm{mmol})$ was stirred with benzaldehyde $(4.80 \mathrm{~g}, 45 \mathrm{mmol})$ at $80^{\circ} \mathrm{C}$ for $72 \mathrm{~h}$. Purification by flash column chromatography (diethyl ether) afforded $8 \mathbf{b}$ as a yellow solid $\left(2.10 \mathrm{~g}, 76 \%\right.$ ), mp $68-70{ }^{\circ} \mathrm{C}$ (from diethyl ether and hexane). (Found: $\mathrm{C}, 75.25 ; \mathrm{H}, 5.43 ; \mathrm{N}, 8.44$. $\mathrm{C}_{20} \mathrm{H}_{16} \mathrm{~N}_{2} \mathrm{O}\left[\mathrm{H}_{2} \mathrm{O}\right]$ requires: $\left.\mathrm{C}, 75.47 ; \mathrm{H}, 5.66 ; \mathrm{N}, 8.81 \%\right) . \delta_{\mathrm{H}}: 7.73$ $(\mathrm{s}, 1 \mathrm{H}, \mathrm{CH}=\mathrm{N}), 7.68(\mathrm{~m}, 2 \mathrm{H}, \mathrm{Ar}-\mathrm{H}), 7.32(\mathrm{~m}, 5 \mathrm{H}, \mathrm{Ar}-\mathrm{H}), 7.23$ (m, 2H, Ar-H), 7.14 (m, 4H, Ar-H), 6.98 (m, 1H, Ar-H), 6.51 (s, $1 \mathrm{H}, \mathrm{H}-2) . \delta_{\mathrm{C}}: 145.1(\mathrm{C}-8 \mathrm{a}), 137.5\left(\mathrm{C}-1^{\prime}\right), 135.7\left(\mathrm{C}-7^{\prime}\right), 132.5$ $(\mathrm{CH}=\mathrm{N}), 132.5,130.3,130.1,129.9,129.6,129.3,128.7,128.2$, 126.6, 126.0, 124.8, 122.4, 121.9 (Ar-CH), 119.9 (C-4a), 118.8 $(\mathrm{Ar}-\mathrm{CH}), 86.2(\mathrm{C}-2) . \mathrm{IR} / \mathrm{cm}^{-1}: v=34363034,2833,1605,1596$, $1560,1523,1509,1488,1461$.

\section{1-(4-Methoxyphenyl)-2-phenyl-1,2-dihydroquinazolin-3-ium-3-} olate 8c

The oxime 7c (408 mg, $1.7 \mathrm{mmol})$ was stirred with benzaldehyde $(8.4 \mathrm{mmol}, 893 \mathrm{mg})$ for $16 \mathrm{~h}$ at $80{ }^{\circ} \mathrm{C}$. Purification by flash column chromatography (diethyl ether) gave $\mathbf{8 c}$ as a yellow solid (526 mg, 94\%), mp $40^{\circ} \mathrm{C}$ (from diethyl ether). (Found: C, 74.16; $\mathrm{H}, 5.42 ; \mathrm{N}, 7.80 . \mathrm{C}_{21} \mathrm{H}_{18} \mathrm{~N}_{2} \mathrm{O}_{2}\left[0.5 \mathrm{H}_{2} \mathrm{O}\right]$ requires: $\mathrm{C}, 74.33 ; \mathrm{H}, 5.60$; $\mathrm{N}, 8.26 \%) . \delta_{\mathrm{H}}: 7.71(\mathrm{~s}, 1 \mathrm{H}, \mathrm{HC}=\mathrm{N}), 7.61(\mathrm{~m}, 2 \mathrm{H}, \mathrm{Ar}-\mathrm{H}), 7.32(\mathrm{~m}$, $3 \mathrm{H}, \mathrm{Ar}-\mathrm{H}), 7.21(\mathrm{~m}, 1 \mathrm{H}, \mathrm{Ar}-\mathrm{H}), 7.05(\mathrm{~m}, 4 \mathrm{H}, \mathrm{Ar}-\mathrm{H}), 6.87(\mathrm{~m}$, $3 \mathrm{H}, \mathrm{Ar}-\mathrm{H}), 6.37(\mathrm{~s}, 1 \mathrm{H}, \mathrm{CH}), 3.79\left(\mathrm{~s}, 3 \mathrm{H}, \mathrm{OCH}_{3}\right) . \delta_{\mathrm{C}}: 157.3(\mathrm{ArC}-$ O), 138.6, 137.9, 136.3 ( $\mathrm{Ar}-\mathrm{C}), 130.4(\mathrm{Ar}-\mathrm{CH}), 130.1(\mathrm{CH}=\mathrm{N})$, 129.3, 128.7, 126.7, 125.9, 124.9, 121.4 (Ar-CH), 118.9 (C-4a), 117.5, $114.9(\mathrm{Ar}-\mathrm{CH}), 86.6(\mathrm{C}-2), 55.5\left(\mathrm{OCH}_{3}\right) . \mathrm{IR} / \mathrm{cm}^{-1}: v=$ 3409, 3059, 2834, 1584, 1557, 1509, 1487, 1459.

\section{1-(4-Cyanophenyl)-2-phenyl-1,2-dihydroquinazolin-3-ium-3- olate 8d}

The oxime 7d (152 mg, $0.64 \mathrm{mmol})$ was stirred with benzaldehyde ( $340 \mathrm{mg}, 3.2 \mathrm{mmol}$ ) at $80^{\circ} \mathrm{C}$ for $72 \mathrm{~h}$. Purification by flash column chromatography afforded $\mathbf{8 d}$ as a white solid ( $93.6 \mathrm{mg}, 45 \%), \mathrm{mp}$ 220-225 ${ }^{\circ} \mathrm{C}$ (from diethyl ether). (Found: $\mathrm{C}, 77.09 ; \mathrm{H}, 4.62 ; \mathrm{N}$, 12.48. $\mathrm{C}_{21} \mathrm{H}_{17} \mathrm{~N}_{3} \mathrm{O}$ requires: $\left.\mathrm{C}, 77.54 ; \mathrm{H}, 4.62 ; \mathrm{N}, 12.92 \%\right) . \delta_{\mathrm{H}}$ : $7.74(\mathrm{~s}, 1 \mathrm{H}, \mathrm{CH}=\mathrm{N}), 7.60(\mathrm{~m}, 4 \mathrm{H}, \mathrm{Ar}-\mathrm{H}), 7.32(\mathrm{~m}, 4 \mathrm{H}, \mathrm{Ar}-\mathrm{H})$, $7.18(\mathrm{~m}, 5 \mathrm{H}, \mathrm{Ar}-\mathrm{H}), 6.51(\mathrm{~s}, 1 \mathrm{H}, \mathrm{H}-2) . \delta_{\mathrm{C}}: 149.3(\mathrm{C}-8 \mathrm{a}), 135.6$ $\left(\mathrm{C}-1^{\prime}\right), 134.6\left(\mathrm{C}-7^{\prime}\right), 134.2,130.6(\mathrm{Ar}-\mathrm{CH}), 130.0(\mathrm{CH}=\mathrm{N})$, 129.4, 126.8, 126.6, 124.7, 121.7, 120.8, $120.4(\mathrm{Ar}-\mathrm{CH}), 119.0$ $(\mathrm{C}-4 \mathrm{a}$ and $\mathrm{C} \equiv \mathrm{N}), 107.3\left(\mathrm{C}-4^{\prime}\right), 86.1(\mathrm{C}-2) . \mathrm{IR} / \mathrm{cm}^{-1}: v=3396$, 3057, 2216, 1598, 1586, 1556, 1506, 1486.

\section{1-(4-Methoxyphenyl)-2-(4-nitrophenyl)-1,2-dihydroquinazolin- 3-ium-3-olate 8e}

The oxime 7c $(1.3 \mathrm{mmol}, 315 \mathrm{mg})$ was stirred in with 4nitrobenzaldehyde $(980 \mathrm{mg}, 6.6 \mathrm{mmol})$ at $120{ }^{\circ} \mathrm{C}$ for $4 \mathrm{~h}$. Purification by flash column chromatography (diethyl ether$\mathrm{MeOH} ; 9.5: 0.5)$ afforded $8 \mathrm{e}$ as a yellow solid $(0.50 \mathrm{~g}, 100 \%)$, mp $143-145{ }^{\circ} \mathrm{C}$ (diethyl ether and hexane). (Found: $\mathrm{C}, 66.83$; $\mathrm{H}, 4.67 ; \mathrm{N}, 11.08 . \mathrm{C}_{21} \mathrm{H}_{17} \mathrm{~N}_{3} \mathrm{O}_{4}$ requires: $\mathrm{C}, 67.20 ; \mathrm{H}, 4.40 ; \mathrm{N}$, $11.08 \%) . \delta_{\mathrm{H}}: 8.17\left(\mathrm{~d}, 2 \mathrm{H}, J 8.7, \mathrm{H}^{\prime} 9^{\prime}\right.$ and $\left.\mathrm{H}-11^{\prime}\right), 7.86(\mathrm{~d}, 2 \mathrm{H}$, $J$ 8.7, $\mathrm{H}-8^{\prime}$ and $\left.\mathrm{H}-11^{\prime}\right), 7.76(\mathrm{~s}, 1 \mathrm{H}, \mathrm{CH}=\mathrm{N}), 7.24(\mathrm{~m}, 1 \mathrm{H}$, $\mathrm{Ar}-\mathrm{H}), 7.05\left(\mathrm{~d}, 2 \mathrm{H}, J\right.$ 8.0, $\mathrm{H}-2^{\prime}$ and $\left.\mathrm{H}-6^{\prime}\right) 6.99(\mathrm{~m}, 3 \mathrm{H}, \mathrm{Ar}-$ $\mathrm{H}), 6.88\left(\mathrm{~d}, 2 \mathrm{H}, J\right.$ 8.0, $\mathrm{H}-3^{\prime}$ and $\left.\mathrm{H}-5^{\prime}\right) 6.45(\mathrm{~s}, 1 \mathrm{H}, 2-\mathrm{H}), 3.81$ $\left(\mathrm{s}, 1 \mathrm{H}, \mathrm{OCH}_{3}\right) \cdot \delta_{\mathrm{C}}: 158.0(\mathrm{ArC}-\mathrm{O}), 148.9\left(\mathrm{ArC}-\mathrm{NO}_{2}\right), 143.4$ (C-8a), 138.3, 138.2 (Ar-C), 131.2 (Ar-CH), $130.8(\mathrm{CH}=\mathrm{N})$, 128.3, 126.5, 125.1, 124.3, $122.7(\mathrm{Ar}-\mathrm{CH}), 119.4$ (C-4a), 118.7, $115.5(\mathrm{Ar}-\mathrm{CH}), 86.4(\mathrm{C}-2), 56.0\left(\mathrm{OCH}_{3}\right) . \mathrm{IR} / \mathrm{cm}^{-1}: v=3413$, 3059, 2835, 1585, 1557, 1510, 1488, 1459. 


\section{1,2-Bis-(4-methoxyphenyl)-1,2-dihydroquinazolin-3-ium-3-} olate $8 f$

The oxime 7c (266 mg, $1.1 \mathrm{mmol})$ and $p$-methoxybenzaldehyde $(750 \mathrm{mg}, 5.5 \mathrm{mmol})$ were heated at $80{ }^{\circ} \mathrm{C}$. Reaction progress reached its limit after 236 h (68\% oxime consumption). Purification by flash column chromatography (diethyl ether-MeOH; $9.5: 0.5)$ gave $8 \mathbf{f f}(192 \mathrm{mg}, 49 \%)$ as a clear green oil. $\delta_{\mathrm{H}}: 7.68(\mathrm{~s}$, $1 \mathrm{H}, \mathrm{CH}=\mathrm{N}), 7.52(\mathrm{~d}, 1 \mathrm{H}, J$ 8.6, $\mathrm{Ar}-\mathrm{H}), 7.18(\mathrm{~m}, 1 \mathrm{H}, \mathrm{Ar}-\mathrm{H})$, $7.06(\mathrm{~d}, 2 \mathrm{H}, J$ 8.6, Ar-H), $7.00(\mathrm{~d}, 1 \mathrm{H}, J$ 8.3, Ar-H), 6.91 (d, $1 \mathrm{H}, J 7.4, \mathrm{Ar}-\mathrm{H}), 6.88(\mathrm{~m}, 4 \mathrm{H}, \mathrm{Ar}-\mathrm{H}), 6.30(\mathrm{~s}, 1 \mathrm{H}, \mathrm{H}-2), 3.80$, $3.76\left(2 \times \mathrm{OCH}_{3}\right)$.

\section{5,10-Dimethyl-6-phenyl-5,8a,11a,11b-tetrahydropyrrolo $\left[3^{\prime}, 4^{\prime}\right.$ : 4,5]isoxazolo[2,3-c]quinazoline-9,11(6H,10H)-diones 14a, 15a and 16a}

A solution of the nitrone $8 \mathbf{a}(0.5 \mathrm{~g}, 2.1 \mathrm{mmol})$ and $N$-methyl maleimide $(0.24 \mathrm{~g}, 2.1 \mathrm{mmol})$ in DCM $\left(20 \mathrm{~cm}^{3}\right)$ was stirred at $\mathrm{rt}$ for $18 \mathrm{~h}$. The solvent was removed under reduced pressure to give the crude reaction products, the ${ }^{1} \mathrm{H}$ NMR spectral analysis of which indicated the presence of three diastereomeric cycloadducts 14a, 15a and 16a in a $5: 6: 1$ ratio. Purification by flash column chromatography (DCM) followed by crystallisation (diethyl ether and hexane) afforded pure samples of $\mathbf{1 4 a}$ $(0.257 \mathrm{~g}, 37 \%)$ and $15 \mathrm{a}(220 \mathrm{mg}, 32 \%)$, and only a trace amount of the minor isomer 16a was isolated.

Microanalytical data were obtained on a mixed sample of 14a and 15a. (Found: $\mathrm{C}, 67.55 ; \mathrm{H}, 5.42 ; \mathrm{N}, 11.65$. $\mathrm{C}_{20} \mathrm{H}_{19} \mathrm{O}_{3} \mathrm{~N}_{3}\left[0.5 \mathrm{H}_{2} \mathrm{O}\right]$ requires: $\left.\mathrm{C}, 67.03 ; \mathrm{H}, 5.59 ; \mathrm{N}, 11.73 \%\right)$.

14a. White powder, $\mathrm{mp} 220-223{ }^{\circ} \mathrm{C}$ (diethyl ether and hexane). $\delta_{\mathrm{H}}: 7.25(\mathrm{~m}, 4 \mathrm{H}, \mathrm{Ar}-\mathrm{H}), 7.11(\mathrm{~m}, 3 \mathrm{H}, \mathrm{Ar}-\mathrm{H}), 6.76(\mathrm{~m}$, 2H, Ar-H), 5.57 (s, 1H, H-6), 4.77 (d, 1H, J 7.3, H-8a), 4.50 (s, $1 \mathrm{H}, \mathrm{H}-11 \mathrm{~b}), 3.77$ (d, 1H, $J$ 7.3, H-11a), 3.07 (s, 3H, $\mathrm{NCH}_{3}$ ), 3.06 $\left(\mathrm{s}, 3 \mathrm{H}, \mathrm{NCH}_{3}\right) . \delta_{\mathrm{C}}: 175.7(\mathrm{C}=\mathrm{O}), 175.6(\mathrm{C}=\mathrm{O}), 143.1(\mathrm{C}-4 \mathrm{a})$, $137.4\left(\mathrm{C}-1^{\prime}\right), 129.3,129.2,128.9,127.4,127.3(\mathrm{Ar}-\mathrm{CH}), 118.2$ (C-11c), 117.4, 111.2 (Ar-CH), 77.4 (C-6), 75.6 (C-8a), 59.5 (C11b), 56.5 (C-11a), $37.7\left(\mathrm{NCH}_{3}\right), 25.5\left[\mathrm{C}(\mathrm{O}) \mathrm{NCH}_{3}\right] . \mathrm{IR} / \mathrm{cm}^{-1}$ : $v=3460,2919,2839,1786,1698,1603,1577,1508,1448$.

nOeds results for 14a. Irradiation of $\mathrm{H}-8 \mathrm{a}$ caused a $5.4 \%$ enhancement on the signal representing $\mathrm{H}-11 \mathrm{a}$; irradiation of $\mathrm{H}-$ 11a caused enhancements on $\mathrm{H}-8 \mathrm{a}(7.1 \%)$ and on $\mathrm{H}-11 \mathrm{~b}(3.2 \%)$; irradiation of $\mathrm{H}-11 \mathrm{~b}$ caused a $2.3 \%$ enhancement on $\mathrm{H}-11 \mathrm{a}$.

15a. White powder, $\mathrm{mp} 220-225^{\circ} \mathrm{C}$ (from diethyl ether and hexane). $\delta_{\mathrm{H}}: 7.53(\mathrm{~m}, 2 \mathrm{H}, \mathrm{Ar}-\mathrm{H}), 7.38(\mathrm{~m}, 3 \mathrm{H}, \mathrm{Ar}-\mathrm{H}), 7.24(\mathrm{~m}$, $2 \mathrm{H}, \mathrm{Ar}-\mathrm{H}), 6.90(\mathrm{~m}, 2 \mathrm{H}, \mathrm{Ar}-\mathrm{H}), 5.22(\mathrm{~s}, 1 \mathrm{H}, \mathrm{H}-6), 5.12(\mathrm{~s}, 1 \mathrm{H}$, H-11b), 4.77 (d, 1H, J 7.3, H-8a), 3.73 (d, 1H, J 7.3, H-11a), $3.02\left[\mathrm{~s}, 3 \mathrm{H}, \mathrm{C}(\mathrm{O}) \mathrm{NCH}_{3}\right], 2.70\left(\mathrm{~s}, 3 \mathrm{H}, \mathrm{NCH}_{3}\right) . \delta_{\mathrm{C}}: 175.3(\mathrm{C}=\mathrm{O})$, $174.6(\mathrm{C}=\mathrm{O}), 146.5(\mathrm{C}-4 \mathrm{a}), 137.0\left(\mathrm{C}-1^{\prime}\right), 129.0(\mathrm{Ar}-\mathrm{CH}), 128.6$ (C-11c), 128.5, 127.3, 120.7, 119.3, 115.3 (Ar-CH), 77.9 (C6), 75.5 (C-8a), 65.3 (C-11b), 55.7 (C-11a), $36.9\left(\mathrm{NCH}_{3}\right), 25.2$ $\left[\mathrm{C}(\mathrm{O}) \mathrm{NCH}_{3}\right]$.

nOeds results for 15a. Irradiation of $\mathrm{H}-8 \mathrm{a}$ caused a $7.1 \%$ enhancement on the signal representing $\mathrm{H}-11 \mathrm{a}$; irradiation of $\mathrm{H}-$ $11 \mathrm{a}$ caused enhancements on $\mathrm{H}-8 \mathrm{a}(7.3 \%)$ and on $\mathrm{H}-11 \mathrm{~b}(2.1 \%)$; irradiation of $\mathrm{H}-11 \mathrm{~b}$ caused a $2.5 \%$ enhancement on $\mathrm{H}-11 \mathrm{a}$.

\section{0-Methyl-5,6-diphenyl-5,8a,11a,11b-tetrahydropyrrolo[ $3^{\prime}, 4^{\prime}$ : 4,5]isoxazolo[2,3-c]quinazoline-9,11(6H,10H)-diones 14b, 15b and $16 \mathrm{~b}$}

A solution of the nitrone $\mathbf{8 b}(153 \mathrm{mg}, 0.51 \mathrm{mmol})$ and $N$-methyl maleimide (57 mg, $0.51 \mathrm{mmol})$ was stirred in DCM $\left(20 \mathrm{~cm}^{3}\right)$ at rt for $18 \mathrm{~h}$. The solvent was removed under reduced pressure to give the crude reaction products, the ${ }^{1} \mathrm{H}$ NMR spectral analysis of which indicated a mixture of three isomeric cycloadducts $\mathbf{1 4 b}$, 15b and 16b in $2.5: 2: 1$ ratio. Purification by flash column chromatography (DCM) followed by crystallization (diethyl ether and hexane) afforded 14b (119 mg, 55\%), 15b (75 mg, $35 \%)$ and $\mathbf{1 6 b}(24 \mathrm{mg}, 10 \%)$.
Microanalytical and $\mathrm{mp}$ data were obtained on a sample of mixed diastereomers; white powder, $\mathrm{mp} 250-252{ }^{\circ} \mathrm{C}$ (from diethyl ether and hexane). (Found: C, 72.38; H, 5.08; N, 10.07. $\mathrm{C}_{25} \mathrm{H}_{21} \mathrm{~N}_{3} \mathrm{O}_{3}$ requires: $\mathrm{C}, 72.99 ; \mathrm{H}, 5.25 ; \mathrm{N}, 10.50 \%$ ).

14b. $\delta_{\mathrm{H}}: 7.39(\mathrm{~m}, 2 \mathrm{H}, \mathrm{Ar}-\mathrm{H}), 7.28(\mathrm{~m}, 5 \mathrm{H}, \mathrm{Ar}-\mathrm{H}), 7.18(\mathrm{~m}$, $2 \mathrm{H}, \mathrm{Ar}-\mathrm{H}), 7.1(\mathrm{~m}, 4 \mathrm{H}, \mathrm{Ar}-\mathrm{H}), 6.87(\mathrm{~m}, 1 \mathrm{H}, \mathrm{Ar}-\mathrm{H}), 5.99(\mathrm{~s}$, 1H, H-6), 4.85 (d, 1H, J 7.2, H-9a), 4.55 (s, 1H, H-11b), 3.82 $\left(\mathrm{d}, 1 \mathrm{H}, J\right.$ 7.2, H-11a), $3.11\left(\mathrm{~s}, 3 \mathrm{H}, \mathrm{NCH}_{3}\right) . \delta_{\mathrm{C}}: 175.7(\mathrm{C}=\mathrm{O})$, $175.6(\mathrm{C}=\mathrm{O}), 148.0(\mathrm{C}-4 \mathrm{a}), 140.9\left(\mathrm{C}-1^{\prime}\right), 137.9\left(\mathrm{C}-7^{\prime}\right), 129.8$, 129.2, 128.9, 128.5, 127.8, 127.6, 124.5, $123.9(\mathrm{Ar}-\mathrm{CH}), 121.1$ (C-11c), 120.5, 118.1 (Ar-CH), 77.9 (C-6), 75.7 (C-8a), 60.00 (C-11b), 56.4 (C-11a), $25.6\left(\mathrm{NCH}_{3}\right) . \mathrm{IR} / \mathrm{cm}^{-1}: v=3473,2951$, 2834, 1788, 1699, 1595, 1578, 1496.

nOeds results for $\mathbf{1 4 b}$. Irradiation of $\mathrm{H}-8$ a caused a $6.1 \%$ enhancement on the signal representing $\mathrm{H}-11 \mathrm{a}$; irradiation of $\mathrm{H}$ 11a caused enhancements on $\mathrm{H}-8 \mathrm{a}(8.3 \%)$ and on $\mathrm{H}-11 \mathrm{~b}(3.6 \%)$; irradiation of $\mathrm{H}-11 \mathrm{~b}$ caused a $3.0 \%$ enhancement on $\mathrm{H}-11 \mathrm{a}$.

15b. $\delta_{\mathrm{H}}: 7.35(\mathrm{~m}, 3 \mathrm{H}, \mathrm{Ar}-\mathrm{H}), 7.13(\mathrm{~m}, 5 \mathrm{H}, \mathrm{Ar}-\mathrm{H}), 7.00(\mathrm{~m}$, $4 \mathrm{H}, \mathrm{Ar}-\mathrm{H}), 6.88(\mathrm{t}, 1 \mathrm{H}, J$ 7.3, Ar-H), $6.66(\mathrm{~d}, 1 \mathrm{H}, J$ 8.2, $\mathrm{Ar}-\mathrm{H})$, 5.68 (s, 1H, H-6), 5.23 (1H, s, H-11b), $4.84(1 \mathrm{H}, \mathrm{d}, J$ 7.4, H-8a), $3.74(1 \mathrm{H}, \mathrm{d}, J 7.4, \mathrm{H}-11 \mathrm{a}), 3.01\left(3 \mathrm{H}, \mathrm{s}, \mathrm{NCH}_{3}\right) . \delta_{\mathrm{c}}: 175.7(\mathrm{C}=\mathrm{O})$, $174.8(\mathrm{C}=\mathrm{O}), 145.6,144.4\left(\mathrm{C}-1^{\prime}\right.$ and $\left.\mathrm{C}-4 \mathrm{a}\right), 136.8\left(\mathrm{C}-7^{\prime}\right), 130.7$, 130.4, 129.4, 128.6, 128.5, 128.2, 127.8, 126.5 (Ar-CH), 120.2 (C-11c), 120.0, $117.2(\mathrm{Ar}-\mathrm{CH}), 78.9$ (C-6), 76.2 (C-8a), 65.8 (C-11b), 56.2 (C-11a), $25.6\left(\mathrm{NCH}_{3}\right) . \mathrm{IR} / \mathrm{cm}^{-1}: v=3471,3035$, 2922, 1783, 1709, 1603, 1593, 1576, 1491 .

nOeds results for $\mathbf{1 5 b}$. Irradiation of H-6 caused a $5.7 \%$ enhancement on the signal representing $\mathrm{H}-11 \mathrm{~b}$; irradiation of $\mathrm{H}-8 \mathrm{a}$ caused a $6.3 \%$ enhancement on the signal representing $\mathrm{H}-11 \mathrm{a}$; irradiation of $\mathrm{H}-11 \mathrm{a}$ caused enhancements on $\mathrm{H}-8 \mathrm{a}$ $(7.0 \%)$ and on $\mathrm{H}-11 \mathrm{~b}(2.8 \%)$; irradiation of $\mathrm{H}-11 \mathrm{~b}$ caused a $3.9 \%$ enhancement on $\mathrm{H}-6$.

16b. $\delta_{\mathrm{H}}: 7.40(\mathrm{~m}, 2 \mathrm{H}, \mathrm{Ar}-\mathrm{H}), 7.29(\mathrm{~m}, 5 \mathrm{H}, \mathrm{Ar}-\mathrm{H}), 7.08(\mathrm{~m}$, $6 \mathrm{H}, \mathrm{Ar}-\mathrm{H}), 6.75$ (t, 1H, J 6.3, Ar-H), 5.95 (s, 1H, H-6), 4.97 (d, $1 \mathrm{H}, J 8.0, \mathrm{H}-8 \mathrm{a}), 4.70$ (d, 1H, $J 8.0, \mathrm{H}-11 \mathrm{~b}), 4.24$ (dd, $1 \mathrm{H}, J 8.0$ and 8.0, $\mathrm{H}-11 \mathrm{a}), 2.63$ (s, $3 \mathrm{H}, \mathrm{NCH}_{3}$ ).

nOeds results for $16 \boldsymbol{b}$. Irradiation of H-6 caused a $4.1 \%$ enhancement on the signal representing $\mathrm{H}-11 \mathrm{a}$; irradiation of $\mathrm{H}-8 \mathrm{a}$ caused a $9.5 \%$ enhancement on the signal representing $\mathrm{H}-11 \mathrm{a}$; irradiation of $\mathrm{H}-11 \mathrm{a}$ caused enhancements on $\mathrm{H}-8 \mathrm{a}$ $(6.6 \%)$ and on $\mathrm{H}-11 \mathrm{~b}(6.2 \%)$; irradiation of $\mathrm{H}-11 \mathrm{~b}$ caused a $8.5 \%$ enhancement on $\mathrm{H}-11 \mathrm{a}$.

\section{5-(4-Methoxyphenyl)-10-methyl-6-phenyl-5,8a,11a,11b- tetrahydropyrrolo[ $\left.3^{\prime}, 4^{\prime}: 4,5\right]$ isoxazolo $[2,3-c]$ quinazoline- 9,11(6H,10H)-diones $14 \mathrm{c}, 15 \mathrm{c}$ and $16 \mathrm{c}$}

A solution of the nitrone $8 \mathbf{c}(0.30 \mathrm{~g}, 0.91 \mathrm{mmol})$ and $N$-methyl maleimide $(101 \mathrm{mg}, 0.91 \mathrm{mmol})$ in DCM $\left(20 \mathrm{~cm}^{3}\right)$ was stirred at $\mathrm{rt}$ for $18 \mathrm{~h}$. The solvent was removed under reduced pressure to give the crude reaction products, the ${ }^{1} \mathrm{H}$ NMR spectral analysis of which indicated the presence of a mixture of three isomeric cycloadducts $\mathbf{1 4 c}, \mathbf{1 5} \mathbf{c}$ and $\mathbf{1 6 c}$ in a ratio of 4.5 : $2.5: 1$. Purification by flash column chromatography (DCM) followed by crystallisation (diethyl ether and hexane) afforded 14c $(0.175 \mathrm{~g}, 46 \%)$, 15c $(0.102 \mathrm{~g}, 27 \%)$ and $\mathbf{1 6 c}(10 \mathrm{mg}, 3 \%)$.

Microanalytical data were obtained on a mixed sample of $\mathbf{1 4 c}$ and 15c. (Found: C, 69.60; H, 5.38; N, 9.23. $\mathrm{C}_{26} \mathrm{H}_{23} \mathrm{~N}_{3} \mathrm{O}_{4}\left[0.5 \mathrm{H}_{2} \mathrm{O}\right]$ requires: $\mathrm{C}, 69.33 ; \mathrm{H}, 5.33, \mathrm{~N}, 9.33 \%)$.

14c. White powder, $190-192^{\circ} \mathrm{C}$ (from diethyl ether). $\delta_{\mathrm{H}}: 7.37$ $(\mathrm{m}, 2 \mathrm{H}, \mathrm{Ar}-\mathrm{H}), 7.27$ (m, 3H, Ar-H), 7.13 (m, 4H, Ar-H), 6.84 (m, 4H, Ar-H), 5.84 (s, 1H, H-6), 4.84 (d, 1H, J 7.5, H-8a), 4.53 (s, 1H, H-11b), 3.80 (d, 1H, J 7.5, H-11a), 3.79 (s, 3H, $\mathrm{OCH}_{3}$ ), $3.09\left(\mathrm{~s}, 3 \mathrm{H}, \mathrm{NCH}_{3}\right) . \delta_{\mathrm{C}}: 175.4(\mathrm{C}=\mathrm{O}), 175.2(\mathrm{C}=\mathrm{O}), 157.1$ (C-4'), $141.7\left(\mathrm{C}-1^{\prime}\right), 140.1,137.9$ (Ar-C), 128.8, 128.4, 128.2, $127.2,126.8(\mathrm{Ar}-\mathrm{CH}), 119.4(\mathrm{C}-11 \mathrm{c}), 119.0,116.3,114.8(\mathrm{Ar}-$ $\mathrm{CH}), 77.7$ (C-6), 75.4 (C-8a), 59.5 (C-11b), 56.1 (C-11a), 55.5 
$\left(\mathrm{OCH}_{3}\right), 25.2\left(\mathrm{NCH}_{3}\right) . \mathrm{IR} / \mathrm{cm}^{-1}: v=3473,2951,2834,1788$, 1701, 1597, 1576, 1508, 1493.

nOeds results for $14 c$. Irradiation of $\mathrm{H}-8$ a caused a $6.4 \%$ enhancement on the signal representing $\mathrm{H}-11 \mathrm{a}$; irradiation of $\mathrm{H}-11 \mathrm{~b}$ caused a $2.9 \%$ enhancement on $\mathrm{H}-11 \mathrm{a}$.

15c. White powder, $190-195^{\circ} \mathrm{C}$ (from diethyl ether). $\delta_{\mathrm{H}}: 7.38$ (m, 2H, Ar-H), 7.27 (m, 1H, Ar-H), 7.14 (m, 3H, Ar-H), 7.01 $(\mathrm{m}, 1 \mathrm{H}, \mathrm{Ar}-\mathrm{H}), 6.93\left(\mathrm{~d}, 2 \mathrm{H}, J\right.$ 8.8, $\mathrm{H}^{\prime} 3^{\prime}$ and $\left.\mathrm{H}-5^{\prime}\right), 6.92(\mathrm{~m}, 1 \mathrm{H}$, Ar-H), $6.66\left(\mathrm{~d}, 2 \mathrm{H}, J\right.$ 8.8, H-2' and $\left.\mathrm{H}-6^{\prime}\right), 6.35(\mathrm{~d}, 1 \mathrm{H}, J$ 8.3, Ar-H), 5.62 (s, 1H, H-6), 5.26 (s, 1H, H-11b) 4.88 (d, 1H, J 7.2, $\mathrm{H}-8 \mathrm{a}), 3.78$ (d, 1H, J 7.2, H-11a), 3.70 (s, 3H, $\left.\mathrm{OCH}_{3}\right), 3.02$ (s, $3 \mathrm{H}, \mathrm{NCH}_{3}$ ).

nOeds results for 15c. Irradiation of H-6 caused a 5.6\% enhancement on the signal representing $\mathrm{H}-11 \mathrm{~b}$; irradiation of $\mathrm{H}-8 \mathrm{a}$ caused enhancements on $\mathrm{H}-6(2.3 \%)$ and on $\mathrm{H}-11 \mathrm{a}(6.5 \%)$; irradiation of $\mathrm{H}-11 \mathrm{~b}$ caused a $6.7 \%$ enhancement on $\mathrm{H}-6$ and a $2.5 \%$ enhancement on $\mathrm{H}-11 \mathrm{a}$.

16c. Isolated as a white powder, $190-192{ }^{\circ} \mathrm{C}$ (from diethyl ether). $\delta_{\mathrm{H}}$ : $7.39(\mathrm{~m}, 2 \mathrm{H}, \mathrm{Ar}-\mathrm{H}), 7.28(\mathrm{~m}, 3 \mathrm{H}, \mathrm{Ar}-\mathrm{H}), 7.12(\mathrm{~d}$, $2 \mathrm{H}, J$ 9.0, H-3' and H-5') $7.05(\mathrm{~m}, 2 \mathrm{H}, \mathrm{Ar}-\mathrm{H}), 6.93(\mathrm{~m}, 1 \mathrm{H}$, $\mathrm{Ar}-\mathrm{H}), 6.86$ (d, $2 \mathrm{H}, J$ 9.0, $\mathrm{H}-2^{\prime}$ and $\left.\mathrm{H}-6^{\prime}\right) 6.70$ (m, $\left.1 \mathrm{H}, \mathrm{Ar}-\mathrm{H}\right)$, 5.81 (s, 1H, H-6), 4.97 (d, 1H, $J$ 8.0, H-8a), 4.67 (br, d, 1H, $J$ 6.2, $\mathrm{H}-11 \mathrm{~b}), 4.25$ (br, dd, $1 \mathrm{H}, J 8.0$ and $8.0, \mathrm{H}-11 \mathrm{a}), 3.79(\mathrm{~s}, 3 \mathrm{H}$, $\left.\mathrm{OCH}_{3}\right), 2.70$ (s, $3 \mathrm{H}, \mathrm{NCH}_{3}$ ).

nOeds results for 16c. Irradiation of $\mathrm{H}-8$ a caused a $5.6 \%$ enhancement on the signal representing $\mathrm{H}-11 \mathrm{a}$; irradiation of $\mathrm{H}-11 \mathrm{a}$ caused enhancements on $\mathrm{H}-8 \mathrm{a}(7.8 \%)$ and on $\mathrm{H}-11 \mathrm{~b}(1.2 \%)$; irradiation of $\mathrm{H}-11 \mathrm{~b}$ caused a $4.3 \%$ enhancement on $\mathrm{H}-11 \mathrm{a}$.

\section{4-[10-Methyl-9,11-dioxo-6-phenyl-8a,9,10,11,11a,11b- hexahydropyrrolo $\left[3^{\prime}, 4^{\prime}: 4,5\right]$ isoxazolo $[2,3-c] q u i n a z o l i n-5(6 H)-$ yllbenzonitriles 14d, 15d and 16d}

A solution of the nitrone $\mathbf{8 d}(111 \mathrm{mg}, 0.5 \mathrm{mmol})$ and $N$-methyl maleimide $(55 \mathrm{mg}, 0.5 \mathrm{mmol})$ in DCM $\left(10 \mathrm{~cm}^{3}\right)$ was stirred at $\mathrm{rt}$ for $18 \mathrm{~h}$. The solvent was removed under reduced pressure. ${ }^{1} \mathrm{H}$ NMR spectral analysis of the crude product indicated the presence of three isomeric cycloadducts 14d, 15d and 16d in a $1: 0.2: 1$ ratio. Purification by flash column chromatography (DCM) followed by crystallization (diethyl ether and hexane) afforded $14 d(0.11 \mathrm{~g}, 50 \%), \mathbf{1 6 d}(0.067 \mathrm{~g}, 30 \%)$ and only a trace amount of $\mathbf{1 5 d}$.

Microanalytical and $\mathrm{mp}$ data were obtained on a mixed sample of $\mathbf{1 4 d}$ and $\mathbf{1 6 d}$, white powder, decomposes at 270 $280{ }^{\circ} \mathrm{C}$ (from diethyl ether). (Found: $\mathrm{C}, 70.73 ; \mathrm{H}, 4.60 ; \mathrm{N}, 12.51$. $\mathrm{C}_{26} \mathrm{H}_{20} \mathrm{~N}_{4} \mathrm{O}_{3}\left[0.5 \mathrm{H}_{2} \mathrm{O}\right]$ requires: $\mathrm{C}, 70.11 ; \mathrm{H}, 4.72 ; \mathrm{N}, 12.58 \%$ ).

14d. $\delta_{\mathrm{H}}: 7.54\left(\mathrm{~d}, 2 \mathrm{H}, J 8.3, \mathrm{H}-3^{\prime}\right.$ and $\left.\mathrm{H}-5^{\prime}\right), 7.26(\mathrm{~m}, 10 \mathrm{H}$, Ar-H) 7.01 (t, 1H, J 7.7, Ar-H), $6.10(\mathrm{~s}, 1 \mathrm{H}, \mathrm{H}-6), 4.83(\mathrm{~d}, 1 \mathrm{H}, J$ 7.3, H-8a), 4.58 (s, 1H, H-11b), 3.82 (d, 1H, J 7.3, H-11a), 3.12 (s, $3 \mathrm{H}, \mathrm{N}-\mathrm{Me}) . \delta_{\mathrm{C}}: 175.0(\mathrm{C}=\mathrm{O}), 174.7(\mathrm{C}=\mathrm{O}), 152.2(\mathrm{C}-4 \mathrm{a}), 138.2$ $\left(\mathrm{C}-1^{\prime}\right), 136.0\left(\mathrm{C}-7^{\prime}\right), 133.6,129.1,128.9,128.5,127.8,127.0(\mathrm{Ar}-$ $\mathrm{CH}), 122.9(\mathrm{C}-11 \mathrm{c}), 122.8,121.3,119.6(\mathrm{Ar}-\mathrm{CH}), 119.2(\mathrm{C} \equiv \mathrm{N})$, 105.5 (C-4'), 77.6 (C-6), 75.2 (C-8a), 59.6 (C-11b), 56.0 (C-11a), $25.3\left(\mathrm{NCH}_{3}\right) . \mathrm{IR} / \mathrm{cm}^{-1}: v=3473,3042,2947,2231,1789,1701$, 1608, 1600, 1585, 1509, 1493.

nOeds results for $14 d$. Irradiation of $\mathrm{H}-8$ a caused a $6.8 \%$ enhancement on the signal representing $\mathrm{H}-11 \mathrm{a}$; irradiation of $\mathrm{H}$ $11 \mathrm{a}$ caused enhancements on $\mathrm{H}-8 \mathrm{a}\left(7.9^{\%}\right)$ and on $\mathrm{H}-11 \mathrm{~b}(3.1 \%)$; irradiation of $\mathrm{H}-11 \mathrm{~b}$ caused a $2.7 \%$ enhancement on $\mathrm{H}-11 \mathrm{a}$.

16d. $\delta_{\mathrm{H}}: 7.57\left(\mathrm{~d}, 2 \mathrm{H}, J 8.5, \mathrm{H}-3^{\prime}\right.$ and $\left.\mathrm{H}-5^{\prime}\right), 7.37(\mathrm{~m}, 2 \mathrm{H}$, $\mathrm{Ar}-\mathrm{H}), 7.25(\mathrm{~m}, 7 \mathrm{H}, \mathrm{Ar}-\mathrm{H}$, including 7.16, d, J 8.5, H-2' and H-6'), 7.05 (d, 1H, J 7.2, Ar-H), 6.90 (m, 1H, J 3.9, Ar-H), 6.08 (s, 1H, H-6), 4.96 (d, 1H, J 8.2, H-8a), 4.75 (d, 1H, J 8.2, $\mathrm{H}-11 \mathrm{~b}), 4.29$ (dd, $1 \mathrm{H}, J 8.2$ and 8.2, $\mathrm{H}-11 \mathrm{a}), 2.66$ (s, 3H, $\mathrm{NCH}_{3}$ ). $\delta_{\mathrm{C}}$ : $173.0(\mathrm{C}=\mathrm{O}), 172.6(\mathrm{C}=\mathrm{O}), 152.2(\mathrm{C}-4 \mathrm{a}) 139.4\left(\mathrm{C}-1^{\prime}\right), 136.2$ $\left(\mathrm{C}-7^{\prime}\right), 134.0,129.4,129.2,128.9,127.5,122.7,121.3(\mathrm{Ar}-\mathrm{CH})$, $120.7(\mathrm{C}-11 \mathrm{c}), 120.4(\mathrm{Ar}-\mathrm{CH}), 119.6(\mathrm{C} \equiv \mathrm{N}), 105.8\left(\mathrm{C}-4^{\prime}\right), 79.0$ (C-6), 76.8 (C-8a), 58.4 (C-11b), 55.5 (C-11a), $24.9\left(\mathrm{NCH}_{3}\right)$.
Preparation of 10-methyl-6-(4-nitrophenyl)-5-(4-methoxyphenyl)$5,8 a, 11 a, 11 b$-tetrahydropyrrolo $\left[3^{\prime}, 4^{\prime}: 4,5\right]$ isoxazolo $[2,3-$ clquinazoline-9,11(6H,10H)-diones 14e, 15e and 16e

A solution of the nitrone $8 \mathbf{e}(161 \mathrm{mg}, 0.43 \mathrm{mmol})$ and $N$-methyl maleimide (48 $\mathrm{mg}, 0.43 \mathrm{mmol})$ in DCM $\left(10 \mathrm{~cm}^{3}\right)$ was stirred at $\mathrm{rt}$ for $18 \mathrm{~h}$. The solvent was removed under reduced pressure to give a crude product, the ${ }^{1} \mathrm{H}$ NMR spectral analysis of which indicated the presence of three isomeric cycloadducts 14e, 15e and 16e in a ratio of $5: 1: 1$. Purification by flash column chromatography (DCM) followed by crystallisation (diethyl ether and hexane) afforded 14e (0.14 g, 67\%), 15e (0.02 g, 10\%) and $16 \mathrm{e}(0.027 \mathrm{~g}, 13 \%)$.

Microanalytical data were obtained on a mixture of 14e, 15e and 16e. (Found: $\mathrm{C}, 63.19 ; \mathrm{H}, 4.41 ; \mathrm{N}, 11.44$. $\mathrm{C}_{26} \mathrm{H}_{22} \mathrm{~N}_{4} \mathrm{O}_{6}\left[0.5 \mathrm{H}_{2} \mathrm{O}\right]$ requires: $\mathrm{C}, 63.03 ; \mathrm{H}, 4.65 ; \mathrm{N}, 11.52 \%$ ).

14e. Light yellow solid, decomposes at $240{ }^{\circ} \mathrm{C}$ (from diethyl ether and hexane). $\delta_{\mathrm{H}}: 8.15\left(\mathrm{~d}, 2 \mathrm{H}, J 8.6, \mathrm{H}^{-} 9^{\prime}\right.$ and $\left.11^{\prime}\right), 7.60(\mathrm{~d}$, $2 \mathrm{H}, J$ 8.6, H- $8^{\prime}$ and $\left.\mathrm{H}-12^{\prime}\right), 7.12(\mathrm{~m}, 4 \mathrm{H}, \mathrm{Ar}-\mathrm{H}), 6.86(\mathrm{~m}, 4 \mathrm{H}$, $\mathrm{Ar}-\mathrm{H}), 5.91$ (s, 1H, H-6), 4.87 (d, 1H, J 7.2, H-8a), 4.47 (s, 1H, $\mathrm{H}-11 \mathrm{~b}), 3.84$ (d, 1H, J 7.2, H-11a), 3.79 (s, 3H, $\left.\mathrm{OCH}_{3}\right), 3.09$ $\left(\mathrm{s}, 3 \mathrm{H}, \mathrm{NCH}_{3}\right) . \delta_{\mathrm{C}}: 175.4,175.2(\mathrm{C}=\mathrm{O}), 157.8(\mathrm{ArC}-\mathrm{O})$ 148.4, 145.3, 141.4, 140.1 (Ar-C), 128.9, 128.8, 127.7, 127.2, 124.5, 120.3 (Ar-CH), 119.7 (C-11c), 117.3, 115.3 (Ar-CH), 77.6 (C-6), 75.8 (C-8a), 59.9 (C-11b), 56.5 (C-11a), $55.9\left(\mathrm{OCH}_{3}\right)$.

$n$ Oeds results for 14e. Irradiation of $\mathrm{H}-8$ a caused a $6.3 \%$ enhancement on the signal representing $\mathrm{H}-11 \mathrm{a}$; irradiation of $\mathrm{H}-11 \mathrm{~b}$ caused a $3.6 \%$ enhancement on $\mathrm{H}-11 \mathrm{a}$.

15e. Yellow solid, decomposes at $235^{\circ} \mathrm{C}$ (from diethyl ether and hexane). $\delta_{\mathrm{H}}: 8.01\left(\mathrm{~d}, 2 \mathrm{H}, J 8.6, \mathrm{H}-9^{\prime}\right.$ and $\left.\mathrm{H}-11^{\prime}\right), 7.59(\mathrm{~d}$, $2 \mathrm{H}, J$ 8.6, H-9' and $\left.\mathrm{H}-12^{\prime}\right), 7.28(\mathrm{~m}, 1 \mathrm{H}, \mathrm{Ar}-\mathrm{H}), 6.96(\mathrm{~m}, 4 \mathrm{H}$, Ar-H, including $\mathrm{d}$ at $6.94,2 \mathrm{H}, J 8.7, \mathrm{H}-2^{\prime}$ and $\left.\mathrm{H}-6^{\prime}\right), 6.69(\mathrm{~d}$, $2 \mathrm{H}, J$ 8.7, $\mathrm{H}-3^{\prime}$ and $\left.5^{\prime}\right), 6.34(\mathrm{~d}, 1 \mathrm{H}, J$ 8.3, Ar-H), $5.72(\mathrm{~s}, 1 \mathrm{H}$, H-6), 5.30 (s, 1H, H-11b), 4.92 (d, 1H, J 7.3, H-8a), 3.84 (d, $1 \mathrm{H}, J$ 7.3, H-11a), 3.70 (s, $\left.3 \mathrm{H}, \mathrm{OCH}_{3}\right), 3.02\left(\mathrm{~s}, 3 \mathrm{H}, \mathrm{NCH}_{3}\right) . \delta_{\mathrm{C}}$ : 175.5, 174.8 (C=O), 158.5 (ArC-O), 147.9, 146.0, 143.6 (Ar-C), $136.1\left(\mathrm{C}-7^{\prime}\right), 132.1,131.3,128.6,127.6,123.4,120.2(\mathrm{Ar}-\mathrm{CH})$, 119.6 (C-11c), 116.9, $115.0(\mathrm{Ar}-\mathrm{CH}), 78.0$ (C-6), 75.8 (C-8a), 65.6 (C-11b), 55.9 (C-11a), $55.6(\mathrm{OMe}), 25.6(\mathrm{NMe})$.

16e. Isolated as a yellow crystalline solid, decomposes at $248{ }^{\circ} \mathrm{C}$ (from diethyl ether and hexane). $\delta_{\mathrm{H}}: 8.15(\mathrm{~d}, 2 \mathrm{H}, J$ 8.6, H-9' and H-11'), 7.59 (d, 2H, $J$ 8.6, H-8' and H-12'), 7.08 (m, $4 \mathrm{H}, \mathrm{Ar}-\mathrm{H}), 6.88(\mathrm{~m}, 3 \mathrm{H}, \mathrm{Ar}-\mathrm{H}), 6.76(\mathrm{~m}, 1 \mathrm{H}, \mathrm{Ar}-\mathrm{H}), 5.81(\mathrm{~s}$, 1H, H-6), 4.98 (d, 1H, J 8.1, H-8a), 4.66 (br, 1H, H-11b), 4.25 (dd, $1 \mathrm{H}, J 8.1$ and $8.1, \mathrm{H}-11 \mathrm{a}), 3.80\left(\mathrm{~s}, 3 \mathrm{H}, \mathrm{OCH}_{3}\right), 2.71(\mathrm{~s}, 3 \mathrm{H}$, $\left.\mathrm{NCH}_{3}\right) . \delta_{\mathrm{C}}: 172.9,172.9(\mathrm{C}=\mathrm{O}), 157.5(\mathrm{ArC}-\mathrm{O}), 148.3,142.1$, $140.0(\mathrm{Ar}-\mathrm{C}), 129.4,129.3,128.4,126.1,124.3,120.2(\mathrm{Ar}-\mathrm{CH})$, 118.3, 117.7 (Ar-C), 115.3 (Ar-CH), 78.8 (C-6), 77.2 (C-8a), $59.2(\mathrm{C}-11 \mathrm{~b}), 55.9\left(\mathrm{OCH}_{3}\right), 53.8(\mathrm{C}-11 \mathrm{a}), 25.0\left(\mathrm{NCH}_{3}\right)$.

$n$ Oeds results for 16e. Irradiation of $\mathrm{H}-8$ a caused a $6.4 \%$ enhancement on the signal representing $\mathrm{H}-11 \mathrm{a}$; irradiation of $\mathrm{H}-11 \mathrm{~b}$ caused a $2.9 \%$ enhancement on $\mathrm{H}-11 \mathrm{a}$.

\section{Dimethyl 5,6-diphenyl-6,10b-dihydro-5 $H$-isoxazolo[2,3- clquinazoline-1,2-dicarboxylate $17 \mathrm{~b}$ and $(Z)$-3-(1,2-diphenyl- 1,2-dihydroquinazolin-3-ium-3-yl)-1,4-dimethoxy-1,4-dioxo-2- buten-2-olate $18 b$.}

A solution of the nitrone $\mathbf{8 b}$ (226 mg, $0.75 \mathrm{mmol})$ and DMAD $(107 \mathrm{mg}, 0.75 \mathrm{mmol})$ was stirred in DCM $\left(10 \mathrm{~cm}^{3}\right)$ at $\mathrm{rt}$ for $18 \mathrm{~h}$. The solvent was removed under reduced pressure to give a crude product. ${ }^{1} \mathrm{H}$ NMR spectral analysis indicated the formation of two new products, $\mathbf{1 7} \mathbf{b}$ and $\mathbf{1 8 b}$, in a ratio of $4: 1$. Purification by flash column chromatography (diethyl ether-hexane; $2: 8$, followed by diethyl ether-MeOH; $99: 1)$ gave pure samples of 17b $(0.21 \mathrm{~g}, 63 \%)$ and $\mathbf{1 8 b}(0.046 \mathrm{~g}, 14 \%)$.

17b. Red amorphous solid, $\mathrm{mp}$ 134-136 ${ }^{\circ} \mathrm{C}$ (from methanol). (Found: C, 70.42; H, 4.99; N, 6.38. $\mathrm{C}_{26} \mathrm{H}_{22} \mathrm{~N}_{2} \mathrm{O}_{3}$ 
requires: $\mathrm{C}, 70.58 ; \mathrm{H}, 4.77 ; \mathrm{N}, 6.33 \%) . \delta_{\mathrm{H}}: 7.48(\mathrm{~m}, 2 \mathrm{H}, \mathrm{Ar}-$ $\mathrm{H}), 7.24(\mathrm{~m}, 11 \mathrm{H}, \operatorname{Ar}-\mathrm{H}), 6.77(\mathrm{~m}, 1 \mathrm{H}, \mathrm{Ar}-\mathrm{H}), 6.29(\mathrm{~s}, 1 \mathrm{H}$, $\mathrm{H}-5), 5.59$ (s, 1H, H-10b), 3.80 (s, 3H, $\left.\mathrm{OCH}_{3}\right), 3.74(\mathrm{~s}, 3 \mathrm{H}$, $\left.\mathrm{OCH}_{3}\right) . \delta_{\mathrm{C}}: 163.01(\mathrm{C}=\mathrm{O}), 159.40(\mathrm{C}=\mathrm{O}), 152.53(\mathrm{C}=C-\mathrm{O})$, 148.18, 141.15 (ArC-N), 136.95 (Ar-C), 129.79, 129.06, 128.67, 128.18, 127.22 (Ar-CH), 126.48 (C-10a), 123.39, 122.01, 121.70, $119.19(\mathrm{Ar}-\mathrm{CH}), 111.63(\mathrm{C}=\mathrm{C}-\mathrm{O}), 78.70(\mathrm{C}-5), 61.80(\mathrm{C}-10 \mathrm{~b})$, $53.38\left(\mathrm{OCH}_{3}\right), 52.09\left(\mathrm{OCH}_{3}\right)$.

18b. Red crystalline solid, mp $128-130{ }^{\circ} \mathrm{C}$ (from diethyl ether and methanol). (Found: $\mathrm{C}, 66.85 ; \mathrm{H}, 5.27 ; \mathrm{N}, 6.17$. $\mathrm{C}_{26} \mathrm{H}_{22} \mathrm{~N}_{2} \mathrm{O}_{5}\left[1.5 \mathrm{H}_{2} \mathrm{O}\right]$ requires: $\left.\mathrm{C}, 66.52 ; \mathrm{H}, 5.33 ; \mathrm{N}, 6.10 \%\right) . \delta_{\mathrm{H}}$ : $8.66(\mathrm{~s}, 1 \mathrm{H}, \mathrm{H}-4), 7.45(\mathrm{~m}, 6 \mathrm{H}, \mathrm{Ar}-\mathrm{H}), 7.30(\mathrm{~m}, 6 \mathrm{H}, \mathrm{Ar}-\mathrm{H})$, 7.09 (s, 1H, H-2), 6.99 (m, 2H, Ar-H), 3.83 (s, 3H, OCH $), 3.46$ $\left(\mathrm{OCH}_{3}\right)$. The sample was insufficiently stable upon standing in $\mathrm{CDCl}_{3}$ to acquire ${ }^{13} \mathrm{C}$ NMR spectral analysis.

\section{(Z)-3-(1,2-Diphenyl-1,2-dihydroquinazolin-3-ium-3-yl)-1,4- dimethoxy-1,4-dioxo-2-buten-2-olate $18 \mathrm{~b}$ from 17b}

A solution of $17 \mathbf{b}(0.020 \mathrm{~g}, 0.04 \mathrm{mmol})$ in $\mathrm{MeOH}\left(5 \mathrm{~cm}^{3}\right)$ was heated at reflux for $18 \mathrm{~h}$. Removal of the solvent afforded the crude product which was purified by flash column chromatography (diethyl ether-MeOH; $99: 1$ ). The ylide $\mathbf{1 8 c}$ was isolated as a red amorphous solid in $70 \%$ yield $(0.007 \mathrm{~g})$, and data agree with those reported above.

(Z)-1,4-Dimethoxy-3-(1-methyl-2-phenyl-1,2-dihydroquinazolin3-ium-3-yl)-1,4-dioxo-2-buten-2-olate 18a and tetramethyl 3-[8a,9,9a-tris(methoxycarbonyl)-5-methyl-5,6,8a,9,9a,9bhexahydrocyclopropa[4,5]isoxazolo[2,3-c]quinazolin-9-yl]-7oxabicyclo[2.2.1]hepta-2,5-diene-1,2,5,6-tetracarboxylate 22

A solution of the nitrone $\mathbf{8 a}(888 \mathrm{mg}, 3.7 \mathrm{mmol})$ and DMAD (530 mg, $3.7 \mathrm{mmol})$ in DCM $\left(30 \mathrm{~cm}^{3}\right)$ was stirred for $18 \mathrm{~h}$ at $\mathrm{rt}$. The solvent was removed under reduced pressure. A ${ }^{1} \mathrm{H}$ NMR spectrum of the crude products indicated the presence of a complex mixture comprising one main product together with a number of more minor products. Purification by flash column chromatography (diethyl ether-hexane; $1: 1$, followed by diethyl ether-methanol; $9: 1)$ gave a pure sample of the major product 18a $(0.75 \mathrm{~g}, 60 \%)$. The cyclopropyl-fused isoxazolidine $22(150 \mathrm{mg}, 5 \%)$ was isolated as colourless crystals that fell out of the first 20 column fractions.

18a. Red crystalline solid, mp $126-128{ }^{\circ} \mathrm{C}$ (from diethyl ether and methanol). (Found: C, 63.55; H, 5.53; N, 7.04. $\mathrm{C}_{21} \mathrm{H}_{20} \mathrm{~N}_{2} \mathrm{O}_{5}\left[\mathrm{H}_{2} \mathrm{O}\right]$ requires: $\left.\mathrm{C}, 63.32 ; \mathrm{H}, 5.53 ; \mathrm{N}, 7.04 \%\right) . \delta_{\mathrm{H}}$ : $8.45(\mathrm{~s}, 1 \mathrm{H}, \mathrm{H}-4), 7.55(\mathrm{~m}, 1 \mathrm{H}, \mathrm{Ar}-\mathrm{H}), 7.34(\mathrm{~m}, 6 \mathrm{H}, \mathrm{Ar}-\mathrm{H}), 6.85$ $(\mathrm{t}, 1 \mathrm{H}, J$ 7.2, Ar-H), $6.74(\mathrm{~s}, 1 \mathrm{H}, \mathrm{H}-2), 6.72(\mathrm{~d}, 1 \mathrm{H}, J 8.7, \mathrm{Ar}-\mathrm{H})$, $3.81\left(\mathrm{~s}, 3 \mathrm{H}, \mathrm{OCH}_{3}\right), 3.61\left(\mathrm{~s}, 3 \mathrm{H}, \mathrm{OCH}_{3}\right), 3.15\left(\mathrm{~s}, 3 \mathrm{H}, \mathrm{NCH}_{3}\right) . \delta_{\mathrm{C}}$ : $172.7\left[\mathrm{C}(\mathrm{O}) \mathrm{CO}_{2} \mathrm{CH}_{3}\right], 168.4\left(\mathrm{CO}_{2} \mathrm{CH}_{3}\right), 164.7\left(\mathrm{CO}_{2} \mathrm{CH}_{3}\right), 161.1$ (C-4), 147.0 (C-8a), $140.1(\mathrm{Ar}-\mathrm{CH}), 134.5\left(\mathrm{C}-1^{\prime}\right), 133.2,130.6$, 129.2, 126.7, $119.7(\mathrm{Ar}-\mathrm{CH}), 114.8(\mathrm{C}-4 \mathrm{a}) 113.1(\mathrm{Ar}-\mathrm{CH}) 102.2$ $\left(\mathrm{N}^{+}-\mathrm{C}^{-}\right), 79.2(\mathrm{C}-2), 52.3\left(\mathrm{OCH}_{3}\right), 51.3\left(\mathrm{OCH}_{3}\right), 36.9\left(\mathrm{NCH}_{3}\right)$. $\mathrm{IR} / \mathrm{cm}^{-1}: v=3496,3428,3064,2944,1735,1660,1627,1593$, 1554, 1522, 1494.

22. Colourless crystalline solid characterised by X-ray crystallographic analysis. (Found: C, 58.01; H, 4.94; N, 3.55. $\mathrm{C}_{39} \mathrm{H}_{38} \mathrm{~N}_{2} \mathrm{O}_{17}$ requires: $\left.\mathrm{C}, 58.06 ; \mathrm{H}, 4.71 ; \mathrm{N}, 3.47 \%\right) . \delta_{\mathrm{H}}: 7.38$ (m, 2H, Ar-H), $7.28(\mathrm{~m}, 3 \mathrm{H}, \mathrm{Ar}-\mathrm{H}), 7.20(\mathrm{~m}, 2 \mathrm{H}, \mathrm{Ar}-\mathrm{H}), 6.76$ (m, 2H, Ar-H), 5.38 (s, 1H, H-2), 5.10 (s, 1H, H-4c), 3.93, 3.91, $3.79,3.70,3.67,3.53,3.54,3.51\left(8 \times \mathrm{OCH}_{3}\right)$.

X-Ray crystal structure determination of 22. Data were collected at 150(2) K on a Bruker SMART 1000 diffractometer. The structure was solved (in the centrosymmetric space group $\left.P 2_{1} / n\right)$ by direct methods and refined by full-matrix leastsquares on $F^{2}$ using the SHELXTL suite of programs. ${ }^{39}$ All the non-hydrogen atoms were refined with anisotropic atomic displacement parameters, and the hydrogen atoms were inserted
Table 1 Crystal data and structure refinement for $\mathbf{2 2}$

\begin{tabular}{ll}
\hline Identification code & tomas 1 \\
Empirical formula & $\mathrm{C}_{39} \mathrm{H}_{38} \mathrm{~N}_{2} \mathrm{O}_{17}$ \\
Formula weight & 806.71 \\
$T / \mathrm{K}$ & $150(2)$ \\
$\lambda / \AA$ & 0.71073 \\
Crystal system & Monoclinic \\
Space group & $P 2_{1} / n$ \\
$a / \AA$ & $11.1011(12)$ \\
$b / \AA$ & $21.952(2)$ \\
$c / \AA$ & $15.9307(17)$ \\
$a /{ }^{\circ}$ & 90 \\
$\beta /^{\circ}$ & $103.285(2)$ \\
$\gamma /{ }^{\circ}$ & 90 \\
$V / \AA^{3}$ & $3778.2(7)$ \\
$Z$ & 4 \\
$D_{\mathrm{c}} / \mathrm{Mg} \mathrm{m}^{-3}$ & 1.418 \\
$\mu / \mathrm{mm}^{-1}$ & 0.113 \\
$F(000)$ & 1688 \\
Crystal size/mm & $0.43 \times 0.22 \times 0.03$ \\
Crystal description & Colourless lath \\
$\theta$ range for data collection $/^{\circ}$ & 1.61 to 25.00 \\
Index ranges & $-13 \leqslant h \leqslant 11$ \\
& $-16 \leqslant k \leqslant 26$ \\
Reflections collected & $-16 \leqslant l \leqslant 18$ \\
Independent reflections & 18415 \\
Completeness to $\theta=25.00^{\circ}(\%)$ & $6642\left(R_{\text {int }}=0.0488\right)$ \\
Absorption correction & 99.8 \\
Max. and min. transmission & Semi-empirical from equivalents \\
Refinement method & 1.000000 and 0.824622 \\
Data/restraints/parameters & Full-matrix least-squares on $F^{2}$ \\
Goodness-of-fit on $F^{2}$ & $6642 / 0 / 523$ \\
Final $R$ indices $[I>2 \sigma(I)]$ & 1.010 \\
$R$ indices (all data) & $R_{1}=0.0475, w R_{2}=0.1027$ \\
Largest diff. peak and hole/e $\AA^{-3}$ & $R_{1}=0.0958, w R_{2}=0.1226$ \\
& 0.267 and -0.251 \\
&
\end{tabular}

at calculated positions using a riding model. Details of the data collection and structure refinement are given in Table 1.

CCDC reference number 282677. For crystallographic data in CIF or other electronic format see DOI: $10.1039 / \mathrm{b} 511998 \mathrm{~g}$.

\section{Dimethyl 6-(4-methoxyphenyl)-5-phenyl-6,10b-dihydro-5 $H$ - isoxazolo[2,3-c]quinazoline-1,2-dicarboxylate $17 \mathrm{c}$ and (Z)-1,4-dimethoxy-3-[1-(4-methoxyphenyl)-2-phenyl-1,2- dihydroquinazolin-3-ium-3-yl]-1,4-dioxo-2-buten-2-olate 18c}

A solution of the nitrone $8 \mathrm{c}(0.846 \mathrm{~g}, 2.6 \mathrm{mmol})$ and DMAD $(0.365 \mathrm{~g}, 2.6 \mathrm{mmol})$ in DCM $\left(20 \mathrm{~cm}^{3}\right)$ was stirred at $\mathrm{rt}$ for $18 \mathrm{~h}$. The solvent was removed under reduced pressure to give the crude reaction products, the ${ }^{1} \mathrm{H}$ NMR spectral analysis of which indicated the formation of two new products, $17 \mathbf{c}$ and $18 \mathbf{c}$, in a ratio of $4: 1$. Trace amounts of other undetermined products were also evident. Purification by flash column chromatography (diethyl ether-hexane; $1: 1$, followed by diethyl ether- $\mathrm{MeOH}$; 9 : 1) gave pure samples of $17 \mathrm{c}(449 \mathrm{mg}, 32 \%)$ and $\mathbf{1 8 c}$ ( $278 \mathrm{mg}, 24 \%$ ). The structure of the latter is confirmed by X-ray crystallographic analysis (see below).

17c. Red powder, $\mathrm{mp} 109-112^{\circ} \mathrm{C}$ (from diethyl ether and hexane). (Found: $\mathrm{C}, 68.31 ; \mathrm{H}, 5.15 ; \mathrm{N}, 5.90 . \mathrm{C}_{27} \mathrm{H}_{22} \mathrm{~N}_{2} \mathrm{O}_{5}$ requires: $\mathrm{C}, 68.64 ; \mathrm{H}, 5.08 ; \mathrm{N}, 5.93 \%) . \delta_{\mathrm{H}}: 7.50(\mathrm{~m}, 2 \mathrm{H}, \mathrm{Ar}-$ $\mathrm{H}), 7.24(\mathrm{~m}, 6 \mathrm{H}, \mathrm{Ar}-\mathrm{H}) 7.16\left(\mathrm{~d}, 2 \mathrm{H}, J 8.9, \mathrm{H}-2^{\prime}\right.$ and $\left.\mathrm{H}-6^{\prime}\right)$, $7.04(\mathrm{~m}, 1 \mathrm{H}, \mathrm{Ar}-\mathrm{H}), 6.85\left(\mathrm{~d}, 2 \mathrm{H}, J 8.9, \mathrm{H}-3^{\prime}\right.$ and $\left.\mathrm{H}^{-} 5^{\prime}\right), 6.73$ $(\mathrm{m}, 1 \mathrm{H}, \mathrm{Ar}-\mathrm{H}), 6.16(\mathrm{~s}, 1 \mathrm{H}, \mathrm{H}-5), 5.55(\mathrm{~s}, 1 \mathrm{H}, \mathrm{H}-10 \mathrm{~b}), 3.84$ (s, $\left.3 \mathrm{H}, \mathrm{OCH}_{3}\right), 3.80\left(\mathrm{~s}, 3 \mathrm{H}, \mathrm{OCH}_{3}\right), 3.75\left(\mathrm{~s}, 3 \mathrm{H}, \mathrm{OCH}_{3}\right) . \delta_{\mathrm{C}}: 163.1$ $(\mathrm{C}=\mathrm{O}), 159.6(\mathrm{C}=\mathrm{O}), 156.5\left(\mathrm{ArC}-\mathrm{OCH}_{3}\right)$ 152.5, 141.9, 141.2 $(2 \times \operatorname{ArC}-\mathrm{N}$ and $\mathrm{C}=C-\mathrm{O}) 137.1\left(\mathrm{C}-7^{\prime}\right), 129.1,128.7,128.3$, $127.0(\mathrm{Ar}-\mathrm{CH}), 125.5(\mathrm{C}-10 \mathrm{a}), 124.7,120.9,118.9,115.1(\mathrm{Ar}-$ $\mathrm{CH}), 111.9(\mathrm{C}=\mathrm{C}-\mathrm{O}), 78.8(\mathrm{C}-5), 61.4(\mathrm{C}-10 \mathrm{~b}), 55.9\left(\mathrm{OCH}_{3}\right)$, $53.6\left(\mathrm{OCH}_{3}\right), 52.3\left(\mathrm{OCH}_{3}\right)$.

18c. Red crystalline solid. (Found: $\mathrm{C}, 66.43 ; \mathrm{H}, 5.19 ; \mathrm{N}, 5.62$. $\mathrm{C}_{27} \mathrm{H}_{22} \mathrm{~N}_{2} \mathrm{O}_{5}\left[\mathrm{H}_{2} \mathrm{O}\right]$ requires: $\left.\mathrm{C}, 66.12 ; \mathrm{H}, 5.31 ; \mathrm{N}, 5.71 \%\right) . \delta_{\mathrm{H}}$ : $8.57(\mathrm{~s}, 1 \mathrm{H}, \mathrm{CH}=\mathrm{N}), 7.45(\mathrm{~m}, 4 \mathrm{H}, \mathrm{Ar}-\mathrm{H}), 7.25(\mathrm{~m}, 4 \mathrm{H}, \mathrm{Ar}-\mathrm{H})$, 
$6.93(\mathrm{~m}, 4 \mathrm{H}, 3 \times \mathrm{Ar}-\mathrm{H}, \mathrm{H}-2), 6.81(\mathrm{~d}, 1 \mathrm{H}, J$ 8.1, $\mathrm{Ar}-\mathrm{H}), 3.82$ $\left(\mathrm{s}, 3 \mathrm{H}, \mathrm{OCH}_{3}\right), 3.81\left(\mathrm{~s}, 3 \mathrm{H}, \mathrm{OCH}_{3}\right), 3.52\left(\mathrm{~s}, 3 \mathrm{H}, \mathrm{OCH}_{3}\right) . \delta_{\mathrm{C}}$ : $172.9\left[\mathrm{C}(\mathrm{O}) \mathrm{CO}_{2} \mathrm{CH}_{3}\right], 168.4\left(\mathrm{CO}_{2} \mathrm{CH}_{3}\right), 164.6\left(\mathrm{COCH}_{3}\right), 159.3$ $\left(\mathrm{ArC}-\mathrm{OCH}_{3}\right), 158.7$ (C-4), 145.7 (C-8a), 138.6 (Ar-CH), 135.7, 135.6 (C-1' and $\left.\mathrm{C}^{\prime} 7^{\prime}\right), 132.4,130.2,129.0,128.2,127.3,121.0$, $116.9(\mathrm{Ar}-\mathrm{CH}), 116.6(\mathrm{C}-4 \mathrm{a}), 115.7(\mathrm{Ar}-\mathrm{CH}), 103.3\left(\mathrm{~N}^{+}-\mathrm{C}^{-}\right)$, 80.0 (C-2), 55.6, 52.4, $51.1\left(3 \times \mathrm{OCH}_{3}\right)$.

$\mathrm{X}$-Ray crystal structure determination of 18c. Single crystals were analyzed using a Nonius Kappa CCD diffractometer. Details of the data collection, solutions, and refinements are given in Table 2. The model was solved and subsequently refined using full-matrix least-squares in SHELXL-97. ${ }^{44}$

CCDC reference number 282676. For crystallographic data in CIF or other electronic format see DOI: $10.1039 / \mathrm{b} 511998 \mathrm{~g}$.

\section{(Z)-1,4-Dimethoxy-3-[1-(4-methoxyphenyl)-2-phenyl-1,2- dihydroquinazolin-3-ium-3-yl]-1,4-dioxo-2-buten-2-olate 18c from $17 \mathrm{c}$}

A solution of $17 \mathrm{c}(0.020 \mathrm{~g}, 0.04 \mathrm{mmol})$ in $\mathrm{MeOH}\left(10 \mathrm{~cm}^{3}\right)$ was stirred at $\mathrm{rt}$ for $18 \mathrm{~h}$. Removal of the solvent afforded the crude product which was purified by flash column chromatography (diethyl ether-MeOH; 99: 1). The ylide 18c was isolated as a red amorphous solid in $50 \%$ yield $(0.010 \mathrm{~g})$; data agree with those reported above.

\section{Dimethyl 6-(4-cyanophenyl)-5-phenyl-6,10b-dihydro-5 $\mathrm{H}$ - isoxazolo[2,3-c]quinazoline-1,2-dicarboxylate 17d}

The nitrone 8d (0.267 g, $0.82 \mathrm{mmol})$ and DMAD (117 mg, $0.82 \mathrm{mmol})$ were stirred in DCM $\left(10 \mathrm{~cm}^{3}\right)$ at $\mathrm{rt}$ for $18 \mathrm{~h}$. The solvent was removed under reduced pressure to leave a red solid, the ${ }^{1} \mathrm{H}$ NMR spectrum of which revealed the presence of a single product, 8d which was purified by crystallization.

17d. Red amorphous solid $(0.384 \mathrm{~g}, 100 \%), \mathrm{mp} 78-83{ }^{\circ} \mathrm{C}$ (from diethyl ether and hexane). (Found: $\mathrm{C}, 66.75 ; \mathrm{H}, 4.72 ; \mathrm{N}$, 8.63. $\mathrm{C}_{27} \mathrm{H}_{21} \mathrm{~N}_{3} \mathrm{O}_{5}\left[\mathrm{H}_{2} \mathrm{O}\right]$ requires: $\mathrm{C}, 66.80 ; \mathrm{H}, 4.74 ; \mathrm{N}, 8.66 \%$ ).

Table 2 Crystal data and structure refinement for $\mathbf{1 8 c}$

\begin{tabular}{|c|c|}
\hline Identification code & $\mathrm{k} 04 \mathrm{pma} 2$ \\
\hline Empirical formula & $\mathrm{C}_{27} \mathrm{H}_{26} \mathrm{~N}_{2} \mathrm{O}_{7}$ \\
\hline Formula weight & 490.50 \\
\hline$T / \mathrm{K}$ & $150(2)$ \\
\hline$\lambda / \AA$ & 0.71073 \\
\hline Crystal system & Triclinic \\
\hline Space group & $P \overline{1}$ \\
\hline$a / \AA$ & $11.2500(2)$ \\
\hline$b / \AA$ & $11.2910(2)$ \\
\hline$c / \AA$ & $11.7080(2)$ \\
\hline$a /^{\circ}$ & $76.459(1)$ \\
\hline$\beta /^{\circ}$ & $64.710(1)$ \\
\hline$\gamma /{ }^{\circ}$ & $64.809(1)$ \\
\hline$V / \AA^{3}$ & $1214.28(4)$ \\
\hline$Z$ & 2 \\
\hline$D_{\mathrm{c}} / \mathrm{Mg} \mathrm{m}^{-3}$ & 1.342 \\
\hline$\mu / \mathrm{mm}^{-1}$ & 0.098 \\
\hline$F(000)$ & 516 \\
\hline Crystal size $/ \mathrm{mm}$ & $0.20 \times 0.15 \times 0.15$ \\
\hline$\theta$ range for data collection $/^{\circ}$ & 3.72 to 27.53 \\
\hline Index ranges & $\begin{array}{l}-14 \leqslant h \leqslant 14 \\
-14 \leqslant k \leqslant 14 \\
-15 \leqslant l \leqslant 15\end{array}$ \\
\hline Reflections collected & 21369 \\
\hline Independent reflections & $5534\left(R_{\mathrm{int}}=0.0399\right)$ \\
\hline Reflections observed $(>2 \sigma)$ & 4325 \\
\hline Data completeness & 0.987 \\
\hline Absorption correction & None \\
\hline Refinement method & Full-matrix least-squares on $F^{2}$ \\
\hline Data/restraints/parameters & $5534 / 3 / 335$ \\
\hline Goodness-of-fit on $F^{2}$ & 1.028 \\
\hline Final $R$ indices $[I>2 \sigma(I)]$ & $R_{1}=0.0634, w R_{2}=0.1751$ \\
\hline $\mathrm{R}$ indices (all data) & $R_{1}=0.0837, w R_{2}=0.1938$ \\
\hline Largest diff. peak and hole/e $\AA^{-3}$ & 0.672 and -0.480 \\
\hline
\end{tabular}

$\delta_{\mathrm{H}}: 7.57(\mathrm{~d}, 2 \mathrm{H}, J$ 8.7, H-9' and H-11') $7.45(\mathrm{~m}, 2 \mathrm{H}, \mathrm{Ar}-\mathrm{H})$, $7.24(\mathrm{~m}, 8 \mathrm{H}, \mathrm{Ar}-\mathrm{H}), 6.90\left(\mathrm{dd}, 1 \mathrm{H}, J 6.5\right.$ and $\left.6.5, \mathrm{H}-4^{\prime}\right), 6.43(\mathrm{~s}$, $1 \mathrm{H}, \mathrm{H}-5), 5.67$ (s, 1H, H-10b), 3.80 (s, 3H, $\mathrm{OCH}_{3}$ ), 3.74 (s, 3H, $\left.\mathrm{OCH}_{3}\right)$.

\section{Preparation of dimethyl 6-(4-methoxyphenyl)-5-(4-nitrophenyl)- 6,10b-dihydro-5 $H$-isoxazolo[2,3-c]quinazoline-1,2- dicarboxylate 17e}

A solution of the nitrone $8 \mathrm{e}(107 \mathrm{mg}, 0.29 \mathrm{mmol})$ and DMAD (41 $\mathrm{mg}, 0.29 \mathrm{mmol})$ in DCM $\left(10 \mathrm{~cm}^{3}\right)$ was stirred at $\mathrm{rt}$ for $18 \mathrm{~h}$. The solvent was removed under reduced pressure. A ${ }^{1} \mathrm{H}$ NMR spectrum of the crude product revealed the presence of only one product, the cycloadduct 17e. Purification by flash column chromatography (diethyl ether-hexane; $1: 1$ ) provided a pure sample of $17 \mathbf{e}(118 \mathrm{mg}, 79 \%)$.

17e. Red amorphous solid, decomposes at $220^{\circ} \mathrm{C}$ (from diethyl ether and hexane). (Found: $\mathrm{C}, 61.67 ; \mathrm{H}, 4.47 ; \mathrm{N}, 7.72$. $\mathrm{C}_{27} \mathrm{H}_{23} \mathrm{~N}_{3} \mathrm{O}_{8}\left[0.5 \mathrm{H}_{2} \mathrm{O}\right]$ requires: $\left.\mathrm{C}, 61.60 ; \mathrm{H}, 4.56 ; \mathrm{N}, 7.98 \%\right) . \delta_{\mathrm{H}}$ : $8.14\left(\mathrm{~d}, 2 \mathrm{H}, J 8.6, \mathrm{H}-3^{\prime}\right.$ and $\left.\mathrm{H}-5^{\prime}\right), 7.71\left(\mathrm{~d}, 2 \mathrm{H}, J 8.6, \mathrm{H}-2^{\prime}\right.$ and H-6'), 7.23 (m, 1H, Ar-H), 7.13 (d, 2H, $J$ 8.8, H-7' and $\left.\mathrm{H}-11^{\prime}\right)$, $7.06(\mathrm{~m}, 2 \mathrm{H}, \mathrm{Ar}-\mathrm{H}), 6.87\left(\mathrm{~d}, 2 \mathrm{H}, J\right.$ 8.8, H- $8^{\prime}$ and $\left.\mathrm{H}-10^{\prime}\right), 6.78$ (m, 1H, Ar-H), 6.19 (s, 1H, H-5), 5.55 (s, 1H, H-10b), 3.84 (s, $\left.3 \mathrm{H}, \mathrm{OCH}_{3}\right), 3.81\left(\mathrm{~s}, 3 \mathrm{H}, \mathrm{OCH}_{3}\right), 3.75\left(\mathrm{~s}, 1 \mathrm{H}, \mathrm{OCH}_{3}\right) . \delta_{\mathrm{C}}: 160.1$, $159.3\left(\mathrm{CO}_{2} \mathrm{CH}_{3}\right), 156.83\left(\mathrm{ArC}-\mathrm{OCH}_{3}\right), 148.29,144.66,141.50$, $140.90(3 \times \mathrm{ArC}-\mathrm{N}$ and $\mathrm{C}=\mathrm{C}-\mathrm{O}), 129.44,129.16\left(\mathrm{C}-1^{\prime}\right.$ and $\mathrm{Ar}-$ CH) $127.28(\mathrm{Ar}-\mathrm{CH}), 125.51$ (C-10a), 124.51, 124.30, 121.65, 119.67, 115.32, 117.71 (Ar-CH), $111.80(C=\mathrm{C}-\mathrm{O}) 78.54(\mathrm{C}-5)$ 61.79 (C-10b), 55.94, 53.65, $52.33\left(\mathrm{OCH}_{3}\right)$.

\section{Acknowledgements}

We acknowledge the support of Fingal County Council (T. McC.) and NUI Maynooth.

\section{References}

1 F. Heaney, S. Bourke, D. Cunningham and P. McArdle, J. Chem. Soc., Perkin Trans. 2, 1998, 547-560.

2 F. Heaney, J. Fenlon, P. McArdle and D. Cunningham, Org. Biomol. Chem., 2003, 1, 1122-1132.

3 F. Heaney and C. O’Mahony, J. Chem. Soc., Perkin Trans. 1, 1998, $341-350$.

4 E. Kowalczyk, J. Wojciechowski and R. Pakula, PL 162851, 1994.

5 M. Anzini, A. Garofalo and S. Vomero, Heterocycles, 1989, 29, 147787.

6 G. E. Field, W. J. Zally and L. H. Sternbach, J. Org. Chem., 1965, 30, 3957-3959.

7 A. Kovendi and M. Kircz, Chem. Ber., 1965, 98, 1049-1059.

8 D. W. Combs, R. Falotico and D. M. Ritchie, US Pat., 4963554, 1990.

9 H. Gnichtel and B. P. Gau, Liebigs Ann. Chem., 1982, 2223-2230.

10 D. Korbonits and P. Kolonits, J. Chem. Soc., Perkin Trans. 1, 1986, 2163-2168.

11 H. Moehrle and J. Lessel, Z. Naturforsch., B: Chem. Sci., 1992, 47, 1333-1340.

12 H. Moehrle and J. Lessel, Chem. Ber., 1992, 125, 1843-1849.

13 H. Moehrle and J. Lessel, Arch. Pharm. (Weinheim, Ger.), 1990, 323, 405-410.

14 H. Mohrle and M. Jeandree, Z. Naturforsch., B: Chem. Sci., 1999, 54, 1577-1588.

15 H. Moehrle and M. Jeandree, Z. Naturforsch., B: Chem. Sci., 1997, 52, 72-78.

16 J. S. Baum, M. E. Condon and D. A. Shook, J. Org. Chem., 1987, 52, 2983-2988.

17 M. Kuroboshi, Y. Waki and H. Tanaka, J. Org. Chem., 2003, 68, 3938-3942.

18 R. I. Fryer, J. V. Earley, N. W. Gilman and W. Zally, J. Heterocycl. Chem., 1976, 13, 433-437.

19 S. Atmaram, A. R. Forrester, M. Gill, R. J. Napier and R. H. Thomson, Acta Chem. Scand. Ser. B, 1982, 36, 641-647.

20 G. F. Field, W. J. Zally and L. H. Sternbach, J. Am. Chem. Soc., 1967, 89, 332-338.

21 E. Lopez-Calle, M. Keller and W. Eberbach, Eur. J. Org. Chem., 2003, 1438-1453. 
22 C. Roemming and P. Kolsaker, Acta Chem. Scand. Ser. B, 1978, 32, 679-682.

23 J. J. D’Amico, B. R. Stults, P. G. Ruminski and K. V. Wood, J. Heterocycl. Chem., 1983, 20, 1283-1286.

24 J. Breu, K.-J. Range, P. Ribel, A. Weber and J. Sauer, Acta Crystallogr., Sect. C, 1996, 52, 2053-2056.

25 G. D. Reid, D. J. Whittaker, W. Roth, B. J. Whitaker, M. J. I. Williamson and G. S. Beddard, Photochem. Photobiol. Sci., 2003, 2, 585-590.

26 R. Grigg, J. F. Malone, T. Mongkolaussavaratana and S. Thianpatanagul, Tetrahedron, 1989, 45, 3849-3862.

27 L. Toupet and Y. Delugeard, Acta Crystallogr., Sect. B, 1979, 35, 1935-1936.

28 J. P. Freeman, Chem. Rev., 1983, 83, 241-261.

29 R. Huisgen and K. Niklas, Heterocycles, 1984, 22, 21-26.

30 A. Liguori, R. Ottana, G. Romeo, G. Sindona and N. Uccella, Heterocycles, 1988, 27, 1365-1376.

31 N. Khan and D. A. Wilson, J. Chem. Res., Synop., 1984, 150-151.

32 E. Lopez-Calle and W. Eberbach, J. Chem. Soc., Chem. Commun., 1994, 301-302.

33 E. LeGoff and R. B. LaCount, Tetrahedron Lett., 1967, 23332335.
34 M. B. Hocking and F. W. van der Voort Maarschalk, Can. J. Chem., 1993, 71, 1873-1889.

35 A. P. Marchand, S. P. Reddy, R. Sharma and G. V. R., Tetrahedron, 1993, 49, 987-994.

36 N. A. Akmanova, K. F. Sagitdinova and E. S. Balenkova, Khim. Geterotsikl. Soedin., 1982, 1192-1194.

37 J. E. C. Baldwin, K. Jin and L. I. Kruse, Tetrahedron, 1985, 41, 5241-5260.

38 J. P. Freeman, D. J. Duchamp, C. G. Chidester, G. Slomp, J. Szmuszkovicz and M. Raban, J. Am. Chem. Soc., 1982, 104, 1380.

39 G. M. Sheldrick, SHELXTL, version 6.12, Bruker AXS, Madison, Wisconsin, USA, 2001.

40 T. Sugasawa, H. Hamana, T. Toyoda and M. Adachi, Synthesis, 1979, 99-100.

41 G. Calestani, R. Leardini, H. McNab, D. Nanni and G. Zanardi, J. Chem. Soc., Perkin Trans. 1, 1998, 1813-1824.

42 G. Jones, B. D. Long and M. P. Thorne, J. Chem. Soc., Perkin Trans. 2, 1992, 903-913.

43 A. K. Colter, P. Plank, J. P. Bergsma, R. Lahti, A. A. Quesnel and A. G. Parsons, Can. J. Chem., 1984, 62, 1780-1784.

44 G. M. Sheldrick, SHELXL-97. A computer program for refinement of crystal structures, University of Göttingen, Germany, 2004. 